\title{
Brain charts for the human lifespan
}

Bethlehem, R.A.I. ${ }^{1},{ }^{2} \#$, Seidlitz J. ${ }^{3},{ }^{4} \#$, White, S.R. ${ }^{5},{ }^{6} \#$, Vogel, J.W. ${ }^{7},{ }^{3}$, Anderson, K.M. ${ }^{8}$, Adamson C. ${ }^{9}{ }^{10}$, Adler S. ${ }^{11}$, Alexopoulos, G.S. ${ }^{12}$, Anagnostou E. ${ }^{13},{ }^{14}$, Areces-Gonzalez A. ${ }^{15},{ }^{16}$, Astle, D.E. ${ }^{17}$, Auyeung B. ${ }^{18},{ }^{1}$, Ayub M. ${ }^{19}$, Ball G. ${ }^{9},{ }^{20}$, Baron-Cohen S. ${ }^{1},{ }^{21}$, Beare R. ${ }^{9},{ }^{10}$, Bedford, S.A. ${ }^{1}$, Benegal $\mathrm{V}^{22}$, Beyer F. ${ }^{23}$, Bin Bae J. ${ }^{24}$, Blangero J. ${ }^{25}$, Blesa Cábez M. ${ }^{26}$, Boardman, J.P. ${ }^{26}$, Borzage M. ${ }^{27}$, Bosch-Bayard, J.F..$^{28}{ }^{29}$, Bourke N. ${ }^{30}$, Calhoun, V.D. ${ }^{31}$, Chakravarty, M.M. ${ }^{32}{ }^{29}$, Chen C. ${ }^{33}$, Chertavian C. ${ }^{3}$, Chetelat G. ${ }^{34}$, Chong, Y.S. ${ }^{35},{ }^{36}$, Cole, J.H. ${ }^{37}{ }^{38}$, Corvin A. ${ }^{39}$, Costantino M. ${ }^{40}{ }^{41}$, Courchesne E. ${ }^{42},{ }^{43}$, Crivello F. ${ }^{44}$, Cropley, V.L. ${ }^{45}$, Crosbie J. ${ }^{46}$, Crossley N. ${ }^{47}{ }^{48}$, Delarue M. ${ }^{34}$, Delorme R. ${ }^{49}{ }^{50}$, Desrivieres S. ${ }^{51}$, Devenyi G ${ }^{52},{ }^{53}$, Di Biase, M.A. ${ }^{45}{ }^{54}$, Dolan R. $.^{55},{ }^{56}$, Donald, K.A..$^{57}$, Donohoe G. ${ }^{58}$, Dunlop K. ${ }^{59}$, Edwards, A.D. ${ }^{60}{ }^{61}$, Elison, J.T. ${ }^{62}$, Ellis, C.T. ${ }^{8}$, Elman, J.A..$^{63}$, Eyler L. ${ }^{64},{ }^{65}$, Fair, D.A. ${ }^{62}$, Feczko E. ${ }^{62}$, Fletcher, P.C. ${ }^{66},{ }^{67}$, Fonagy P. ${ }^{68}$, Franz, C.E. ${ }^{69}$, Galan-Garcia L. ${ }^{70}$, Gholipour A. ${ }^{71}$, Giedd J. ${ }^{72}{ }^{73}$, Gilmore, J.H. ${ }^{74}$, Glahn, D.C. ${ }^{75}{ }^{76}$, Goodyer I. ${ }^{5}$, Grant, P.E. ${ }^{77}$, Groenewold, N.A. ${ }^{78}{ }^{79}$, Gunning, F.M. ${ }^{12}$, Gur, R.E. ${ }^{3}{ }^{80}$, Gur, R.C. ${ }^{3},{ }^{81}$, Hammill, C.F. ${ }^{46},{ }^{82}$, Hansson O. ${ }^{83}{ }^{84}$, Hedden T. ${ }^{85},{ }^{86}$, Heinz A. ${ }^{87}$, Henson, R.N. ${ }^{17},{ }^{88}$, Heuer K ${ }^{89},{ }^{90}$, Hoare J. ${ }^{91}$, Holla B. ${ }^{92}$, Holmes, A.J. ${ }^{93}$, Holt R. ${ }^{1}$, Huang H. ${ }^{94},{ }^{95}$, Im K. ${ }^{96},{ }^{97}$, Ipser J. ${ }^{98}$, Jack Jr, C.R. ${ }^{99}$, Jackowski, A.P. ${ }^{100},{ }^{101}$, Jia T. ${ }^{102},{ }^{103},{ }^{104}$, Johnson, K.A. ${ }^{105},{ }^{106},{ }^{76},{ }^{107}$, Jones, P.B. ${ }^{5},{ }^{108}$, Jones, D.T. ${ }^{109},{ }^{110}$, Kahn R. ${ }^{111},{ }^{112}$, Karlsson H. ${ }^{113},{ }^{114}$, Karlsson L. ${ }^{113},{ }^{114}$, Kawashima R. ${ }^{115}$, Kelley, E.A. ${ }^{116}$, Kern S. ${ }^{117}$, Kim K. ${ }^{118},{ }^{119},{ }^{120},{ }^{121}$, Kitzbichler, M.G. ${ }^{5}$, Kremen, W.S. ${ }^{63}$, Lalonde F. ${ }^{122}$, Landeau B. ${ }^{34}$, Lee S. ${ }^{123}$, Lerch J. ${ }^{124},{ }^{125},{ }^{126}$, Lewis, J.D. ${ }^{127}$, Li J. ${ }^{128}$, Liao W. ${ }^{128}$, Liston C. ${ }^{129}$, Lombardo, M.V. ${ }^{130},{ }^{1}$, LV J. ${ }^{45},{ }^{131}$, Lynch C. ${ }^{59}$, Mallard, T.T. ${ }^{132}$, Marcelis M. ${ }^{133}$, Markello, R.D. ${ }^{134}$, Mathias, S.R. ${ }^{75}$, Mazoyer B. ${ }^{44},{ }^{135}$, McGuire P. ${ }^{136}$, Meaney, M.J. ${ }^{137}$, Mechelli A. ${ }^{136}$, Medic N. ${ }^{5}$, Misic B. ${ }^{134}$, Morgan, S.E. ${ }^{138},{ }^{139},{ }^{140}$, Mothersill D. ${ }^{141},{ }^{142},{ }^{143}$, Nigg J. ${ }^{144}$, Ong, M.Q.W. ${ }^{145}$, Ortinau C. ${ }^{146}$, Ossenkoppele R. ${ }^{147},{ }^{148}$, Ouyang M. ${ }^{94}$, Palaniyappan L. ${ }^{149}$, Paly L. ${ }^{34}$, Pan, P.M. ${ }^{150}{ }^{151}$, Pantelis C. ${ }^{152},{ }^{153},{ }^{154}$, Park, M.M. ${ }^{155}$, Paus T. ${ }^{156}{ }_{157}{ }^{15}$, Pausova Z. ${ }^{46},{ }^{158}$, Paz-Linares D. ${ }^{15},{ }^{159}$, Pichet Binette A. ${ }^{160},{ }^{161}$, Pierce K. ${ }^{162}$, Qian X. ${ }^{145}$, Qiu J. ${ }^{163}$, Qiu A. ${ }^{164}$, Raznahan A. ${ }^{122}$, Rittman T. ${ }^{165}$, Rodrigue A. ${ }^{75}$, Rollins, C.K. ${ }^{166},{ }^{167}$, Romero-Garcia R. ${ }^{5}{ }^{168}$, Ronan L. ${ }^{5}$, Rosenberg, M.D. ${ }^{169}$, Rowitch, D.H. ${ }^{170}$, Salum, G.A. ${ }^{171},{ }^{172}$, Satterthwaite, T.D. ${ }^{7}{ }^{3}$, Schaare, H.L. ${ }^{173},{ }^{174}$, Schachar, R.J. ${ }^{46}$, Schultz, A.P. ${ }^{105},{ }^{175},{ }^{76}$, Schumann G. ${ }^{176},{ }^{177},{ }^{178}$, Schöll M. ${ }^{179},{ }^{180},{ }^{181}$, Sharp D. ${ }^{30}$, Shinohara, R.T. ${ }^{33},{ }^{182}$, Skoog I. ${ }^{117}$, Smyser, C.D. ${ }^{183}$, Sperling, R.A. ${ }^{105},{ }^{106},{ }^{76}$, Stein, D.J. ${ }^{184}$, Stolicyn A. ${ }^{185}$, Suckling J. ${ }^{5}$, Sullivan G. ${ }^{26}$, Taki Y. ${ }^{115}$, Thyreau B. ${ }^{115}$, Toro R. ${ }^{186},{ }^{90}$, Traut N. ${ }^{186},{ }^{187}$, Tsvetanov, K.A. ${ }^{165},{ }^{188}$, Turk-Browne, N.B. ${ }^{8}{ }^{189}$, Tuulari, J.J. ${ }^{190}{ }^{191},{ }^{192}$, Tzourio C. ${ }^{193}$, Vachon-Presseau É. ${ }^{194},{ }^{195},{ }^{196}$, Valdes-Sosa, M.J. ${ }^{70}$, Valdes-Sosa, P.A. ${ }^{197},{ }^{198}$, Valk, S.L. ${ }^{199}$, van Amelsvoort T. ${ }^{200}$, Vandekar, S.N. ${ }^{201},{ }^{202}$, Vasung L. ${ }^{203}$, Victoria, L.W. ${ }^{12}$, Villeneuve S. ${ }^{161}{ }^{204},{ }^{160}$, Villringer A. ${ }^{23},{ }^{205}$, Vértes, P.E. ${ }^{5}{ }^{206}$, Wagstyl K. ${ }^{207}$, Wang, Y.S. ${ }^{208},{ }^{209}{ }^{210}{ }^{211}$, Warfield, S.K. ${ }^{71}$, Warrier V. ${ }^{1}$, Westman E. ${ }^{212}$, Westwater, M.L. ${ }^{5}$, Whalley, H.C. ${ }^{185}$, Witte, A.V. ${ }^{205},{ }^{23},{ }^{213}$, Yang N. ${ }^{208}{ }^{209},{ }^{210},{ }^{211}$, Yeo B. ${ }^{214},{ }^{215},{ }^{216},{ }^{217}$, Yun H. ${ }^{218}$, Zalesky A. ${ }^{219}$, Zar, H.J. ${ }^{78}$, ${ }^{220}$, Zettergren A. ${ }^{117}$, Zhou, J.H. ${ }^{145},{ }^{221},{ }^{214}$, Ziauddeen $H^{5}{ }^{5}{ }^{222},{ }^{223}$, Zugman A. ${ }^{224},{ }^{225}$, Zuo, X.N. ${ }^{208},{ }^{209},{ }^{210},{ }^{211}{ }^{226}, 3 R-B R A I N *$, AIBL ${ }^{* *}$, Alzheimer's Disease Neuroimaging Initiative ${ }^{* * *}$, Alzheimer's Disease Repository Without Borders Investigators ${ }^{* * *}$, UMN BCP ${ }^{* * * *}$, CALM Team $^{* * * * *}$, Cam-CAN ${ }^{* * * * * *}$, CCNP ${ }^{* * * * * * * *}$, COBRE ${ }^{* * * * * * * *}$, ENIGMA Developmental Brain Age

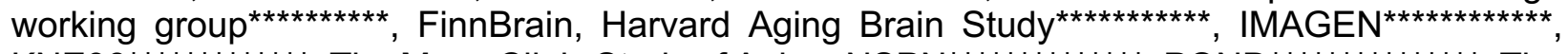
KNE96 $6^{* * * * * * * * * * * *}$, The Mayo Clinic Study of Aging, NSPN ${ }^{* * * * * * * * * * * * *}$, POND $^{* * * * * * * * * * * * * *}$, The PREVENT-AD Research Group, VETSA, Bullmore, E.T. ${ }^{5}$, Alexander-Bloch, A.F. ${ }^{3}{ }^{4} \dagger$

\# equal contribution first authors

$\dagger$ equal contribution senior authors

Corresponding authors:

Richard A.I. Bethlehem, rb643@medschl.cam.ac.uk

Jakob Seidlitz, jakob.seidlitz@pennmedicine.upenn.edu 


\section{Abstract}

Over the past 25 years, neuroimaging has become a ubiquitous tool in basic research and clinical studies of the human brain. However, no reference standards currently exist to quantify individual differences in neuroimaging metrics over the lifespan, in contrast to growth charts for anthropometric traits such as height and weight ${ }^{1}$. Here, we built an interactive resource (www.brainchart.io) to benchmark individual differences in brain morphology, measured from any current or future magnetic resonance imaging (MRI) study, against normative age-related trends. With the goal of basing these reference charts on the largest and most inclusive dataset available, we aggregated 123,984 MRI scans from 101,457 participants in over 100 studies - from 115 days post-conception through 100 postnatal years. Cerebrum tissue volumes and other global or regional MRI metrics were quantified by centile scores, relative to non-linear trajectories ${ }^{2}$, demonstrating concurrent validity with non-MRI brain growth milestones, high stability over longitudinal assessments, and robustness to differences between studies. Brain charts identified previously unreported neurodevelopmental milestones ${ }^{3}$, and centile scores had increased heritability compared to non-centiled MRI phenotypes. Crucially, for clinical purposes ${ }^{4}$, centile scores provided a standardised and interpretable measure of deviation that revealed new patterns of neuroanatomical differences across neurological and psychiatric disorders. In sum, brain charts for the human lifespan are an essential first step towards robust, standardised quantification of deviation from age-related trends in multiple commonly-used neuroimaging phenotypes. Our global collaborative study provides such an anchorpoint for neuroimaging research and will facilitate implementation of quantitative standards in clinical studies.

\section{Main}

First published in the late 18th century ${ }^{1}$, the simple framework of growth charts to quantify developmental change against a reference standard remains a cornerstone of paediatric care. Although a powerful example of 'personalised' or 'precision' medicine, growth charts exist mainly for a small set of anthropometric variables, e.g., height, weight and head circumference. Critically, brain growth and maturation continues well beyond developmental periods covered by anthropometric charts. The lack of brain reference standards is particularly relevant to psychiatric disorders that are increasingly considered to be disorders of neurodevelopment ${ }^{4}$, and arguably represent the single highest current global health burden ${ }^{5}$. Furthermore, preterm infants and those born with congenital conditions - many with psychiatric sequelae - show marked morphological differences during early brain development ${ }^{6,7}$ and even decades later during adulthood ${ }^{7,8}$. With ageing, neurodegeneration and accelerated changes in brain tissue volumes are hallmark signatures of Alzheimer's disease (AD) ${ }^{9}$ and other types of dementia. Modernising the concept of growth charts to generate analogous life-spanning reference charts for the human brain would allow for standardised comparison across samples at scale, while simultaneously advancing our understanding of atypicality by providing benchmark reference points for individual differences in both typical development and ageing.

Such standards for neuroimaging-based reference charts have not yet materialised, likely due to the challenges of integration across studies targeting specific developmental epochs and clinical 
conditions. In particular, the perinatal period is rarely incorporated in lifespan models, despite evidence that early biophysical and molecular processes shape brain growth trajectories ${ }^{10,11}$ and vulnerability to psychiatric conditions ${ }^{3}$. Moreover, case-control comparisons are usually limited to a single disorder despite evidence of shared risk factors and convergent mechanisms, especially in psychiatry ${ }^{12,13}$. Compared to relatively simple anthropometric variables like height or weight, brain morphometrics derived from neuroimaging are more sensitive to methodological variation in scanner platforms, acquisition, and analytic strategy, which severely limits the generalisability of trajectories estimated from individual datasets ${ }^{14}$. Collaborative initiatives spurring collection of large-scale datasets ${ }^{15,16}$, recent advances in neuroimaging data processing ${ }^{17,18}$, and proven statistical frameworks $s^{2,19,20}$ provide the building blocks for comprehensive, life-spanning reference charts of the human brain. Here, we present lifespan models of brain development and ageing that i) robustly capture the normative spectrum of age and sex; ii) identify previously unreported brain growth milestones; iii) increase sensitivity to genetic and early life events; iv) provide standardised effect-size deviations that reveal new patterns of neuroanatomical differences across multiple clinical disorders; and v) represent a global resource for future neuroimaging studies to leverage the benefits of normative benchmarks for precise, quantitative assessment of individual differences in brain structure.

\section{Mapping normative brain growth}

We created brain charts for the human lifespan using generalised additive models for location, scale and shape (GAMLSS) ${ }^{2,19}$, a robust and flexible framework for modelling non-linear growth trajectories recommended by the World Health Organization ${ }^{19}$. Models were fitted to structural MRI data from control subjects for the four main tissue volumes of the cerebrum (total cortical grey matter volume [GMV] and total white matter volume [WMV], total subcortical grey matter volume [sGMV], and total ventricular cerebrospinal fluid volume [Ventricles or CSF]). See Online Methods, Supplementary Table [ST] 1.1-1.7 for details on acquisition, processing and demographics of the dataset. See Supplementary Information [SI] for details regarding GAMLSS model specification and estimation (SI1), image quality control (SI2), model stability and robustness (SI3-4), phenotypic validation against non-imaging metrics (SI3 \& SI5.2), inter-study harmonisation (SI5) and assessment of cohort effects (SI6).

Our models extend previously reported growth curves in multiple ways. Lifespan curves (Fig.1; ST2.1) show an initial strong increase in GMV from mid-gestation onwards, peaking at $5.9_{\mathrm{Cl}}$ Bootstrap:5.8-6.1 years, followed by a near-linear decrease. This peak was observed 2-3 years later than prior reports relying on smaller, more age-restricted samples ${ }^{21,22}$. WMV also increased

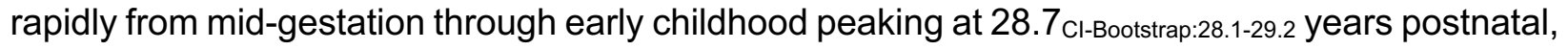
with subsequent accelerated decline in WMV after 50 years. Subcortical GMV showed an intermediate growth pattern compared to GMV and WMV, peaking in adolescence at $14.4_{\mathrm{Cl}}$ Bootstrap:14.0-14.7 years. Both the WMV and SGMV peaks are consistent with prior neuroimaging and postmortem reports ${ }^{23,24}$. In contrast, CSF showed an increase until age 2, followed by a plateau until age 30, and then a slow linear increase that exponentiated in the sixth decade of life. Previously reported growth curves have not generally modelled age-related changes in the variability of brain structure. Age-related variance (Fig.1D), explicitly estimated by GAMLSS, formally quantifies developmental changes in between-subject variability. There was an early 
developmental increase in GMV variability that peaked at 4 years, whereas subcortical volume variability peaked in late adolescence. WMV variability peaked during the fourth decade of life, and CSF was maximally variable at the end of the human lifespan.

\section{A I Aggregated MRI Datasets}
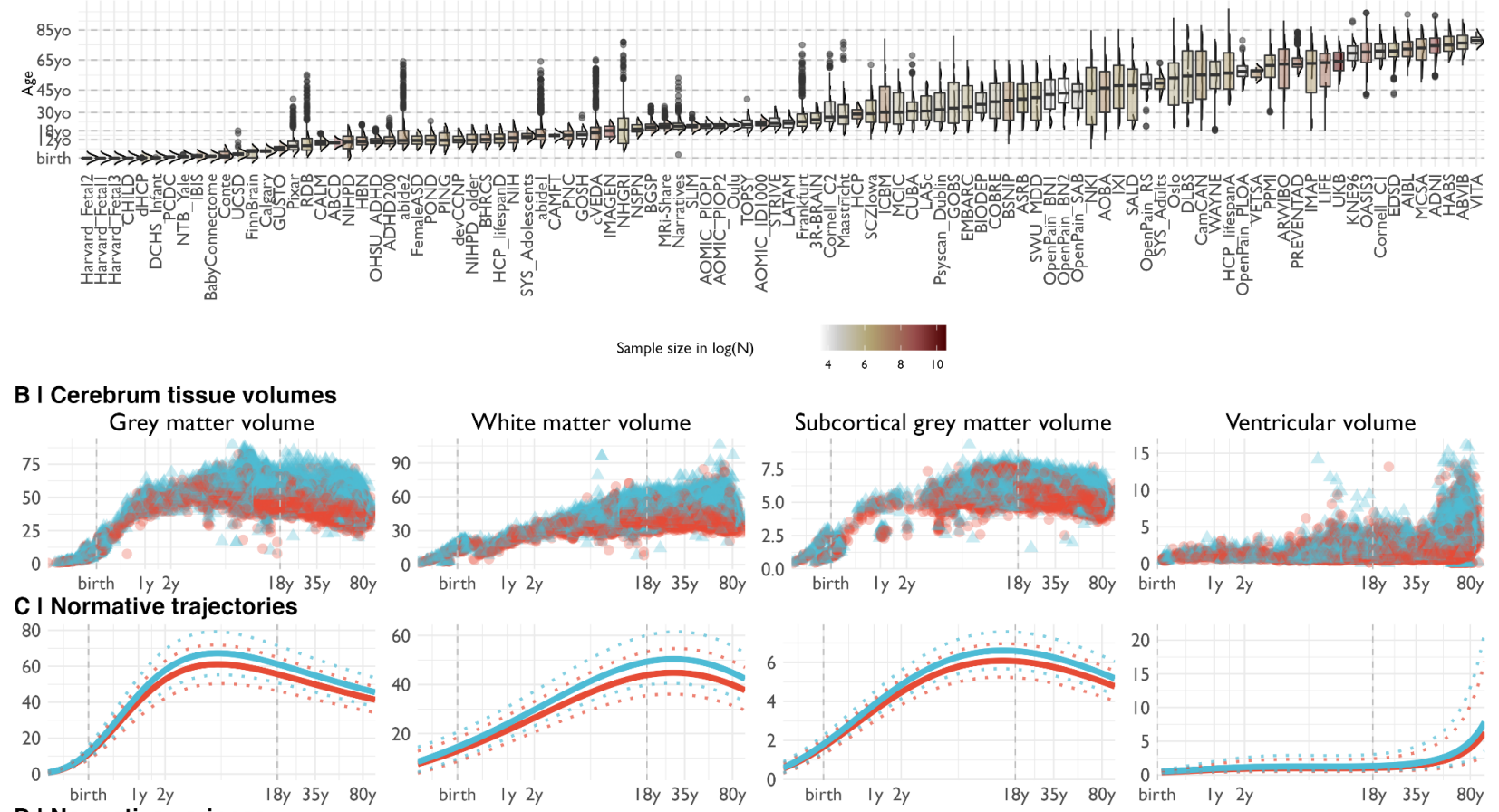

$18 y \quad 35 y \quad 80 y$
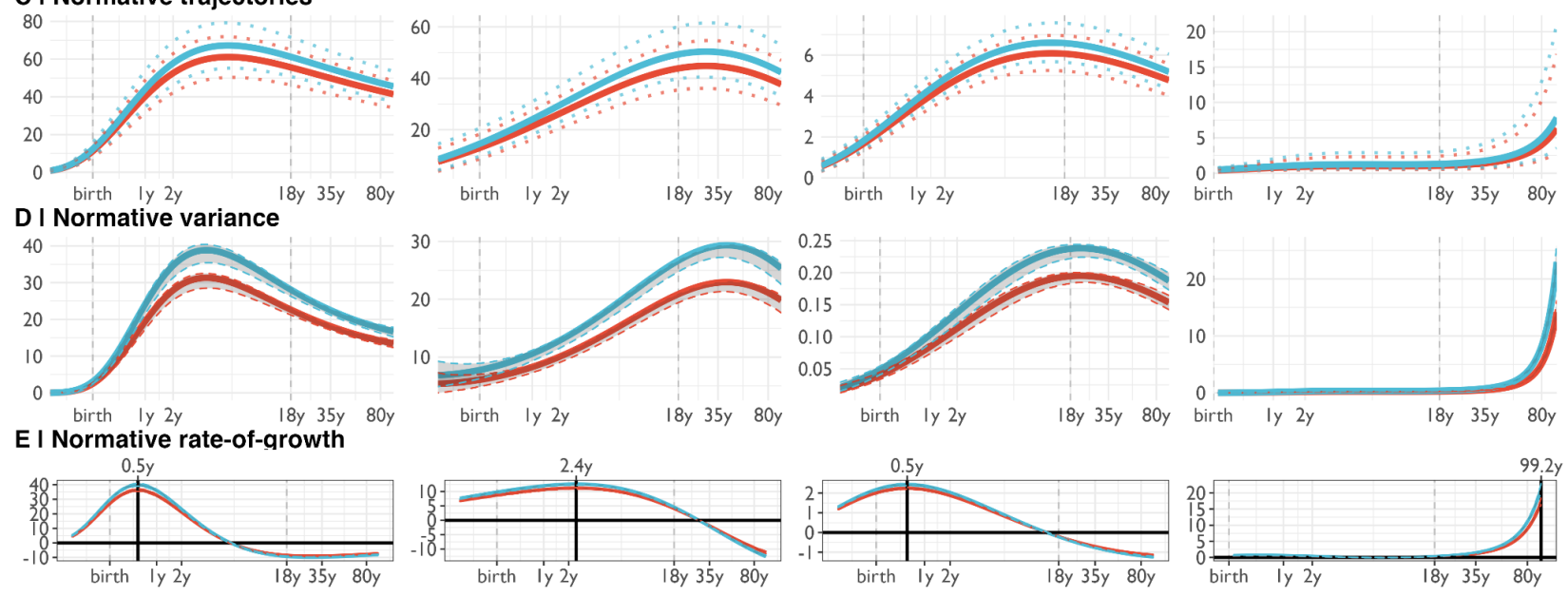

Fig. 1. Human brain charts. A| MRI data were aggregated from 100 primary studies comprising 123,984 scans that collectively spanned the age range from late pregnancy to 100 postnatal years. Box-violin plots show age distributions (log-scaled) for each study coloured by its relative sample-size (log-scaled) B | Noncentiled bilateral cerebrum tissue volumes (right to left: grey matter, white matter, subcortical grey matter and ventricles) are plotted for each cross-sectional control scan, point-coloured by sex, as a function of age (log-scaled). C | Normative brain growth curves, analogous to paediatric growth charts, were estimated by generalised additive modelling for location scale and shape (GAMLSS), accounting for site- and studyspecific batch effects, and stratified by sex (female/male curves coloured red/blue). All four cerebrum tissue volumes demonstrated distinct, non-linear trajectories of their medians and $95 \%$ centile boundaries as a function of age over the life-cycle. Demographics for each cross-sectional sample of healthy controls included in the reference dataset for normative GAMLSS modeling of each MRI phenotype are detailed in ST1.2-1.7. $D$ | Trajectories of median between-subject variability and $95 \%$ confidence intervals for four cerebrum issue volumes were estimated by sex-stratified bootstrapping (1,000 times; see SI3 for details). $E \mid$ Rates of volumetric change across the lifespan for each tissue volume, stratified by sex, were estimated 
by the first derivatives of the median volumetric trajectories. For solid (parenchymal) tissue volumes, the solid horizontal line $(y=0)$ indicates when the volume of each tissue stops growing and starts shrinking; the solid vertical line indicates the age of maximum growth of each tissue. See ST2.1 for all neurodevelopmental milestones and their confidence intervals. Note that $y$-axes in panels $B$-E are scaled in units of $10,000 \mathrm{~mm}^{3}(10 \mathrm{ml})$.

\section{Extended brain MRI phenotypes}

To extend the scope of brain charts beyond the four cerebrum tissue volumes, we used the same GAMLSS modeling approach to estimate normative trajectories for additional MRI phenotypes including other geometric properties at a similar scale (mean cortical thickness and total surface area) and regional volume at each of 34 cortical areas $^{25}$ (Fig.2, SI7-9, ST1-2). We found, as expected, that total surface area closely tracked the development of total cerebrum volume (TCV) across the lifespan (Fig.2A), with both metrics peaking at $\sim 11-12$ years (SA $10.97_{\mathrm{Cl}-\text { Bootstrap:10.42- }}$ 11.51; TCV 12.5cl-Bootstrap:12.14-12.89). In contrast, cortical thickness peaked distinctly early at $1.7_{\mathrm{Cl}}$ Bootstrap:1.3-2.1 years, which reconciles prior observations that cortical thickness increases during the perinatal period ${ }^{26}$ and declines during later development ${ }^{27}$. We also found evidence for regional variability in volumetric neurodevelopmental trajectories. Compared to GMV's peak at 5.9 years, the age of peak regional volume varied considerably - from approximately 2 to 10 years - across 34 cortical areas. Primary sensory regions reached peak volume earliest, and fronto-temporal association cortical areas matured later (Fig.2B; SI8). In general, earlier maturing ventral-caudal regions also showed faster post-peak declines in cortical volume, and later maturing dorsal-rostral regions showed slower post-peak declines (Fig.2B; SI8.2). Notably, this spatial pattern recapitulates a gradient from sensory-to-association cortex that has been previously associated with multiple aspects of brain structure and function ${ }^{28}$.

A I Extended global cortical geometric phenotypes

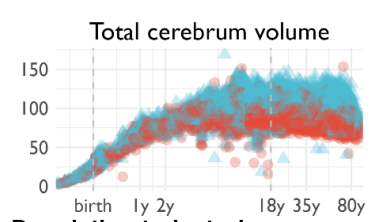

Population trajectories

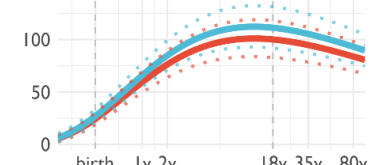

Population variance

Populati
100

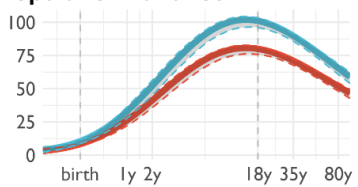

Population rate-of-qrowth
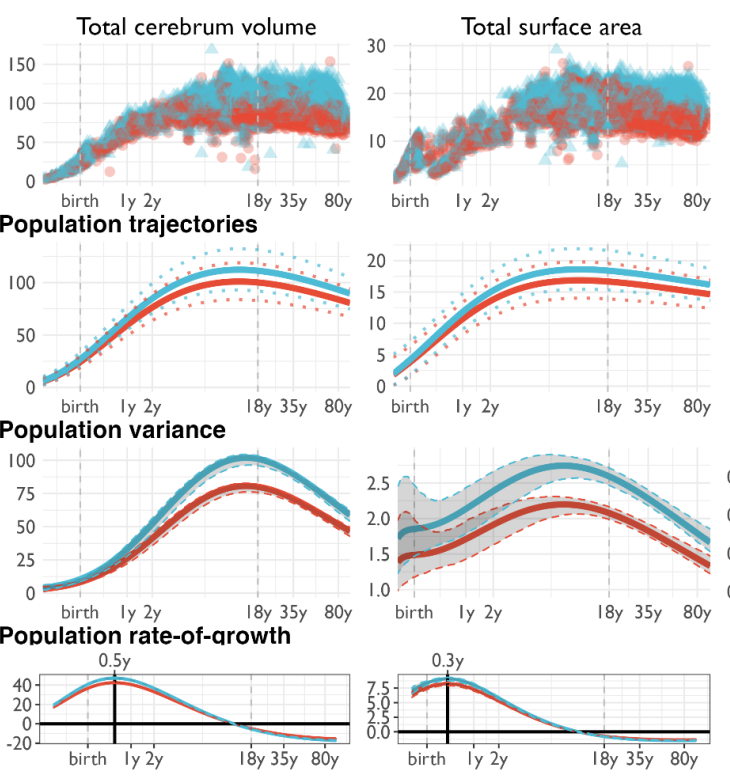

$18 y 35 y \quad 80 y$

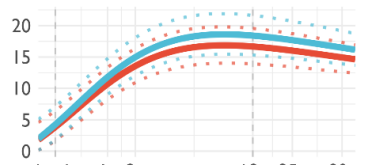

$18 y 35 y \quad 80 y$
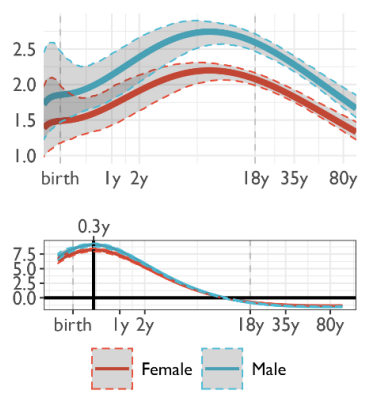

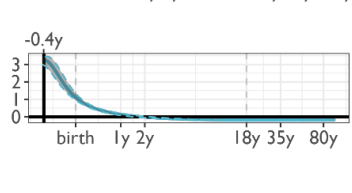

\section{B I Regional phenotypes}

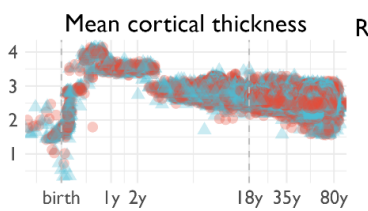

Regional peak volume
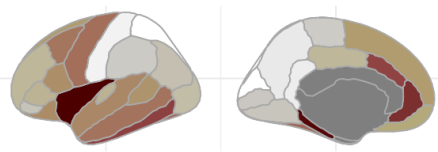

Age (in years)
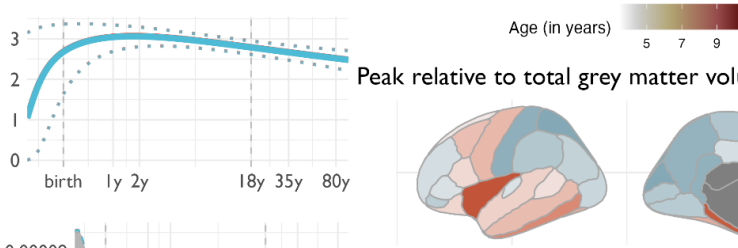

matter volume
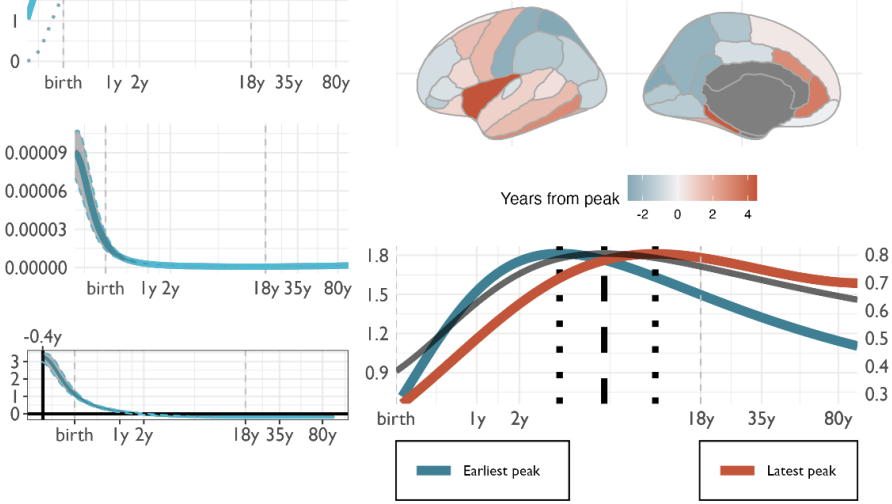
Fig 2. Extended global and regional cortical geometric phenotypes. A | Trajectories for total cerebrum volume (TCV; left column), total surface area (SA; middle column), and mean cortical thickness (CT; right column). For each global cortical geometric MRI phenotype, the following sex-stratified results are shown as a function of age over the life-span, from top to bottom rows: raw, non-centiled data, population trajectories of the median (with 2.5\% and 97\% centiles; dotted lines), between-subject variance (and 95\% confidence intervals), and rate-of-growth (the first derivatives of the median trajectory and $95 \%$ confidence interval). All trajectories are plotted on log-scaled age (x-axis) and $y$-axes are scaled in units of the corresponding MRI metrics $\left(10,000 \mathrm{~mm}^{3}\right.$ for TCV, $10,000 \mathrm{~mm}^{2}$ for SA and $\mathrm{mm}$ for CT). B | Regional variability of cortical volume trajectories for 34 bilateral brain regions as defined in the Desikan Killiany parcellation ${ }^{25}$, averaged across sex (see also SI7-8 for details). From top to bottom panels: cortical map of age at peak regional volume (range 2-10 years); cortical map of age at peak regional volume relative to age at peak GMV (5.9 years), highlighting regions that peak earlier (blue) or later (red) than GMV; and illustrative trajectories for the earliest peaking region (superior parietal lobe) and the latest peaking region (insula), showing the range of regional variability. Regional volume peaks are denoted as dotted vertical lines either side of the global peak denoted as a dashed vertical line in the bottom panel. Left hand y-axis on the bottom panel refers to the earliest peak, the right hand $y$-axis refers to the latest peak, and both are in units of $10,000 \mathrm{~mm}^{3}$.

\section{Developmental milestones}

Neuroimaging milestones are defined by inflection points of the tissue-specific volumetric trajectories (Fig.3; Online Methods). Relative to traditional pubertal age milestones ${ }^{29}$, among the total tissue volumes only GMV peaked before typical pubertal onset, with sGMV peaking midpuberty and WMV peaking in young adulthood (Fig.3). The rate-of-growth (velocity) peaked for

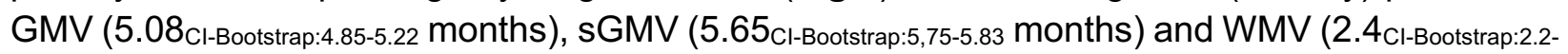
2.6 years) in infancy and early childhood. TCV velocity peaked between the maximum velocity for GMV and WMV at $\sim 7$ months. Two major milestones of TCV and SGMV (peak velocity and size; Fig.3) coincided with the early neonatal and adolescent peaks of height and weight velocity ${ }^{30,31}$. The velocity of mean cortical thickness peaked even earlier, in the prenatal period at $-0.38 \mathrm{Cl}$ Bootstrap:- 0.4 to -0.34 years (relative to birth), corresponding approximately to the second half of pregnancy. This very early peak in cortical thickness velocity has not been reported previously, probably due to the unprecedented aggregation of foetal MRI data allowing precise estimation of early human brain growth in the current study ${ }^{23,32}$. Similarly, the exact timing of the GMV:WMV differentiation in early development, due to changes in myelination and synaptic proliferation ${ }^{33-37}$, has not been clearly demarcated by prior studies. In contrast, lifespan curves indicated an early period of GMV:WMV differentiation, beginning with the switch from WMV to GMV as the dominant tissue compartment in the first month after birth (GMV/WMV ratio=1), and ending when the absolute difference of GMV and WMV peaked around 3 postnatal years (SI9). This epoch of GMV:WMV differentiation encompasses dynamic changes in brain metabolites ${ }^{38}$ (0-3 postnatal months), resting metabolic rate (RMR; minimum $=7$ months, maximum $=4.2$ years $)^{39}$, the typical period of acquisition of motor capabilities and other early paediatric milestones ${ }^{40}$, interneuron migration, and the most rapid change in TCV (Fig.3). 


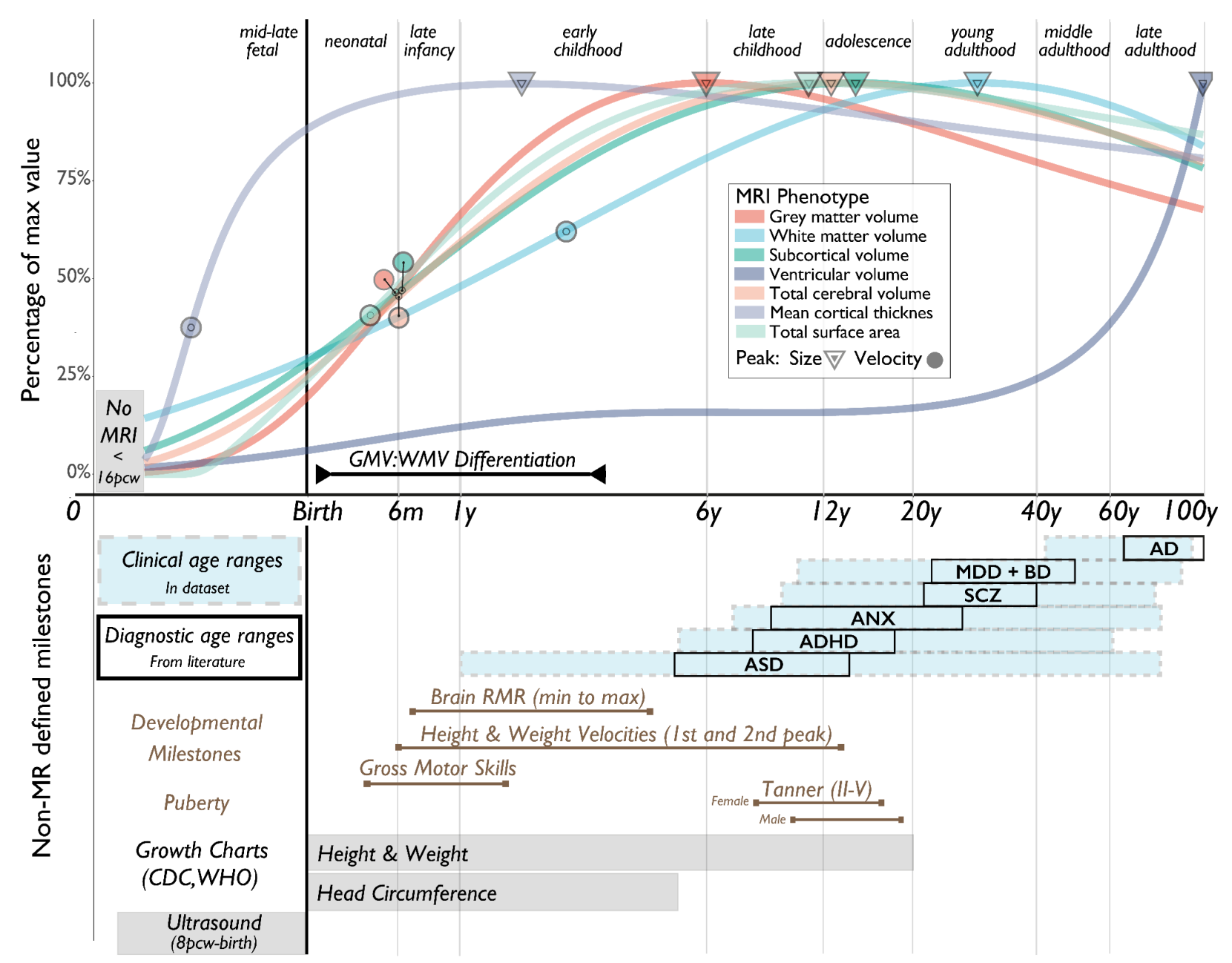

Fig. 3. Neurodevelopmental milestones. Top panel: A graphical summary of the normative trajectories of the median,i.e., 50th centile, for each global MRI phenotype, and key developmental milestones, as a function of age (log-scaled). Circles depict the peak rate-of-growth milestones for each phenotype (defined by the maxima of the first derivatives of the median trajectories; see Fig.1E). Triangles depict the peak volume of each phenotype (defined by the maxima of the median trajectories), definition of GMV:WMV differentiation is detailed in SI9.1. Bottom panel: A graphical summary of additional MRI and non-MRI developmental stages and milestones. From top to bottom: blue shaded boxes denote the age-range of incidence for each of the major clinical disorders represented in the MRI dataset; black boxes denote the age at which these conditions are generally diagnosed as derived from literature ${ }^{41}$ (Online Methods); brown lines represent the normative intervals for developmental milestones derived from non-MRI data, based on previous literature and averaged across males and females (Online Methods); grey bars depict age ranges for existing (WHO and CDC) growth charts of anthropometric and ultrasonographic variables. Across both panels, light grey vertical lines delimit lifespan epochs (labelled above the top panel) previously defined by neurobiological criteria ${ }^{42}$. Abbreviations: resting metabolic rate (RMR), Alzheimer's disease $(A D)$, attention deficit hyperactivity disorder (ADHD), anxiety or phobic disorders (ANX), autism spectrum disorder ( $A S D$, including high-risk individuals with confirmed diagnosis at a later age), major depressive disorder (MDD), bipolar disorder (BD), and schizophrenia (SCZ).

\section{Individualised centile scores in clinical samples}

We computed individualised centile scores that benchmarked each individual scan in the context of normative age-related trends (SI1-6). The clinical diversity of the aggregated dataset allowed 
us to comprehensively investigate case-control differences in individually-specific centile scores. Relative to the control group (CN), there were highly significant differences in centile scores across large $(\mathrm{N}>500)$ diagnostic groups of multiple disorders (Fig.4A; SI10, ST3-4). The pattern of these centile differences varied across tissue types and disorders. Clinical differences in cortical thickness and surface area generally followed the same trend as volume differences (SI10). AD showed the greatest overall difference, with a maximum difference localised to gray matter in biologically female patients (36\% difference from CN median; Fig.4A). In addition, we generated a cumulative deviation metric, the "centile Mahalanobis distance" (CMD), across all brain phenotypes relative to the $\mathrm{CN}$ mean, to summarise a comparative assessment of brain morphology (Fig.4B; SI1.6). Notably, schizophrenia (SCZ) ranked third overall behind AD and mild cognitive impairment (MCl), based on CMD (Fig.4C). Assessment across diagnostic groups, based on profiles of the multiple centile scores and CMD, highlighted shared and distinct patterns across clinical conditions (SI10-11). However, when examining cross-disorder similarity, hierarchical clustering yielded three clusters broadly comprising neurodegenerative, mood/anxiety, and neurodevelopmental disorders (SI11). Overall, these analyses highlight the complementary use-cases for examining both absolute and relative differences in centile scores within and across conventional diagnostic categories.

Between-subject variation in centile scores also showed strong associations with development, early-life events, and shared genetic architecture. Across individual lifespan epochs, CMD was consistently greater in cases relative to controls, irrespective of diagnostic category, with the largest difference found in adolescence and late adulthood across epochs ${ }^{42}$ (SI10.3). Adolescence also represented the greatest period of overlap across diagnostic categories in our dataset and is well-recognised as a period of overall vulnerability for neuropsychiatric disease (Fig.4; SI10-11). Across 5 primary studies covering the lifespan, average centile scores were related to two metrics of premature birth (gestational age at birth: $t=13.164, P<2 \mathrm{e}-16$; birth weight: $t=36.395, P<2 \mathrm{e}-16$; SI12). Centile scores also showed increased twin-based heritability in two independent studies (total $\mathrm{N}=913$ twin-pairs) compared to non-centiled phenotypes (11.8\% mean difference in $\mathrm{h}^{2}$; Fig.4C, SI13). In summary, centile normalisation of brain metrics reproducibly detected case-control differences and genetic effects on brain structure, as well as long-term sequelae of adverse birth outcomes even in the adult brain ${ }^{8}$. 


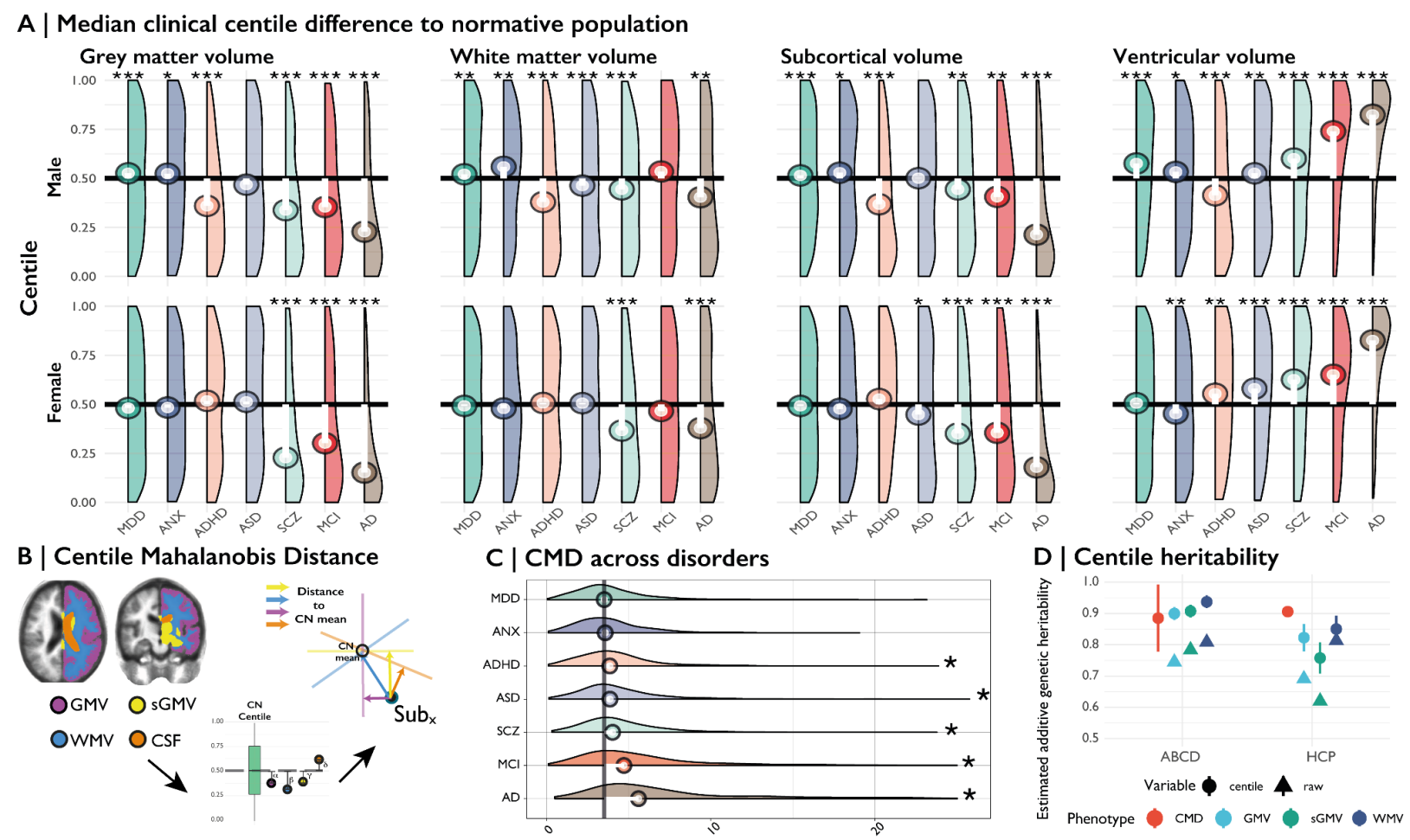

Fig. 4. Case-control differences and heritability of centile scores. A | Centile score distributions for each diagnostic category of clinical cases relative to the control group median (depicted as a horizontal black line). The median deviation of centile scores in each diagnostic category is overlaid as a lollipop plot (white line with circle corresponding to the median centile score for each group of cases). Pairwise tests for significance were based on Monte-Carlo resampling (10,000 permutations) and P-values were adjusted for multiple comparisons using the Benjamini-Hochberg False Discovery Rate (FDR) correction across all possible case-control differences. Only significant deviations from the control group median (with corrected $P<0.001)$ are highlighted with an asterisk. For a complete overview of all pairwise comparisons, see SI10 and ST3. Groups are ordered by their multivariate distance from the control group (see panel C and SI10.3). $B \mid$ The centile Mahalanobis distance (CMD) is a summary metric of multivariate deviation that quantifies the aggregate atypicality of an individual scan in terms of all MRI phenotypes. The schematic shows segmentation of four cerebrum tissue volumes, followed by estimation of univariate centile scores, leading to the orthogonal projection of a single subject (Subx) onto the four principal components of the control group (CN; coloured axes and arrows): the CMD for Sub $b_{x}$ is then the sum of its distances from the CN group mean on all 4 dimensions of the multivariate space. $C \mid$ Probability density plots of CMD across disorders. Vertical black line depicts the median CMD of the control group. Asterisks indicate an FDR-corrected significant difference from the $C N$ group $(P<0.001)$. $D \mid$ Heritability of raw volumetric phenotypes and their centile scores across two twin studies ( $A B C D$ and $H C P)$. All dots have error bars for the standard error, but in some cases these are too narrow to be observed. Abbreviations: control (CN), Alzheimer's disease (AD), attention deficit hyperactivity disorder (ADHD), anxiety or phobia (ANX), autism spectrum disorder (ASD), mild cognitive impairment (MCI), major depressive disorder (MDD), schizophrenia (SCZ); grey matter volume (GMV), subcortical grey matter volume (sGMV), white matter volume (WMV), centile Mahalanobis distance (CMD).

\section{Longitudinal centile changes}

Due to the relative paucity of longitudinal imaging data ( $10 \%$ of the reference dataset), normative models were estimated from single cross-sectional time points. However, the generalisability of cross-sectional models to longitudinal assessment is important for potential clinical utility. Withinsubject variability of centile scores derived from longitudinally repeated scans, measured with 
the interquartile range (IQR; SI1.7), was low across both clinical and control groups (all median $<0.05$ centile), indicating that centile scoring of brain structure was generally stable over time, although there was also some evidence of between-study and cross-disorder differences in within-subject variability (SI14). Notably, individuals who changed diagnostic categories showed small but significant increases in within-subject variability of centile scores (SI14; ST5-6). Withinsubject variability was also slightly higher in younger samples (SI14), which could reflect increased noise due to the technical difficulties associated with scanning younger individuals, but is also consistent with the evidence of increased variability in earlier development observed across other anthropometric traits ${ }^{43}$.

\section{Out-of-sample centile scoring of "new" MRI data}

A key challenge for brain charts is the accurate centile scoring of out-of-sample MRI data, not represented in the normative distribution of trajectories. As such, we carefully evaluated the reliability and validity of brain charts for centile scoring of "new" scans. For each new MRI study, we used maximum likelihood to estimate study-specific statistical offsets from the age-appropriate epoch of the normative trajectory; then we estimated centile scores for each individual in the new study benchmarked against the offset trajectory (Fig.5; SI1.8). Extensive jack-knife and leaveone-study-out (LOSO) analyses indicated that a study size of $N>100$ scans was sufficient for stable and unbiased estimation of out-of-sample centile scores (SI4). Furthermore, out-of-sample centile scores proved highly reliable in multiple test-retest datasets and robust to variations in image processing pipelines (SI4).

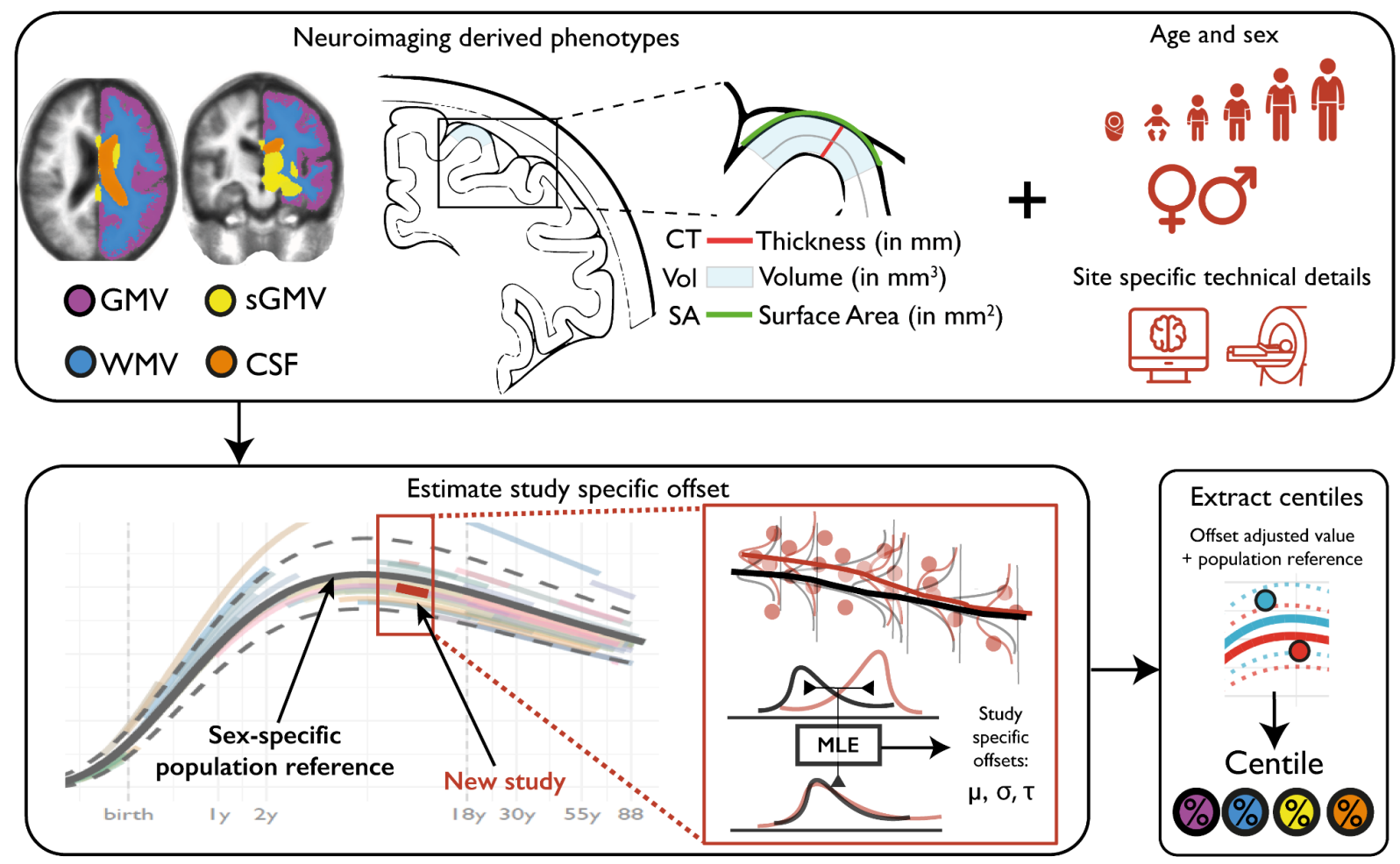

Fig. 5. Schematic overview of brain charts, highlighting methods for out-of-sample centile scoring. Top panel: Brain phenotypes are measured in a reference dataset of MRI scans. GAMLSS modeling is 
used to estimate the relationship between (global) MRI phenotypes and age, stratified by sex, and controlling for technical and other sources of variation between scanning sites and primary studies. Bottom panel: The normative trajectory of the median and confidence interval for each phenotype is plotted as a population reference curve. Out-of-sample data from a new MRI study are aligned to the corresponding epoch of the normative trajectory, using maximum likelihood to estimate the study specific offsets (random effects) for three moments of the underlying statistical distributions: mean $(\mu)$, variance $(\sigma)$, and skewness $(\nu)$ in an age- and sex-specific manner. Centile scores can then be estimated for each scan in the new study, on the same scale as the reference population curve, while accounting for study-specific "batch effects" on technical or other sources of variation (see Sl1.8 for details).

\section{Discussion}

We have aggregated the largest neuroimaging dataset to date to modernise the concept of growth charts for mapping typical and atypical human brain development and ageing. The $\sim 100$ year age range enabled the delineation of milestones and critical periods in brain maturation, revealing an early growth epoch across its constituent tissue classes -- starting before 17 post-conception weeks, when the brain is at $\sim 10 \%$ of its overall size and ending at $\sim 80 \%$ by age 3 . Individual centile scores benchmarked by normative neurodevelopmental trajectories were significantly associated with neuropsychiatric disorders as well as with individual differences in birth outcomes and fluid intelligence (SI5.2 and SI12). Furthermore, imaging-genetics studies ${ }^{44}$ may benefit from the increased heritability of centile scores compared to raw volumetric data (SI13). Perhaps most importantly, GAMLSS modeling enabled harmonisation across technically diverse studies (SI5), and thus leveraged the potential power of aggregating MRI datasets at scale.

The current results also bode well for future progress towards individualised prediction ${ }^{45}$. By providing an age- and sex-normalised metric, centile scores enable trans-diagnostic comparisons between disorders that emerge at different stages of the lifespan (SI10-11). The generally high stability of centile scores across longitudinal measurements also enabled assessment of documented changes in diagnosis such as the transition from $\mathrm{MCl}$ to $\mathrm{AD}$ (SI14), which provides one example of how centile scoring could be clinically useful in quantitatively predicting or diagnosing progressive neurodegenerative disorders. The analogy to paediatric growth charts is not meant to imply a predetermined or immediate application for brain charts in a typical clinical setting. However, our provision of appropriate normative growth charts and on-line tools creates an opportunity to quantify atypical brain structure, precisely benchmarked against age- and sextypical distributions, and thus to enhance diagnostic yield from clinical scans as well as neuroimaging research studies.

Presently, even the current large and diverse dataset is not fully representative of the global population at all ages. For example, foetal, neonatal and mid-adulthood (30-40y) epochs were under-represented (SI17-19); and, as is also common in existing genetic datasets, ethnicity and geography were heavily biased towards European and North American populations. While our statistical modeling approach was designed to mitigate study- or site-specific bias in centile scores, further increasing diversity in MRI research will enable more population-representative normative trajectories ${ }^{46,47}$ that can be expected to improve the accuracy and strengthen the interpretation of centile scores in relation to demographically appropriate norms. Our decision to 
stratify the lifespan models by sex followed the analogous logic of sex-stratifed anthropometric growth charts. Males have larger brain tissue volumes than females in absolute terms (SI16) but this is not indicative of any difference in clinical or cognitive outcome. Future work would also benefit from more detailed and dimensional self-report variables relating to sex and gender ${ }^{48}$.

We have focused primarily on charts of global brain phenotypes, which were measurable in the largest aggregated sample over the widest age range, with the fewest methodological, theoretical and data sharing constraints. However, we have also provided proof-of-concept brain charts for regional grey matter volumetrics, demonstrating plausible heterochronicity of cortical patterning, and illustrating the generalisability of this approach to a more diverse range of fine-grained MRI phenotypes. As ongoing and future efforts provide increasing amounts of high quality MRI data, we predict an iterative process of improved brain charts for the human lifespan, potentially representing multi-modal MRI phenotypes and enabling out-of-sample centile scoring of smaller samples or individual scans. In the hope of facilitating progress in this direction, we have provided interactive tools to explore these statistical models and to derive normalised centile scores for new datasets across the lifespan at www.brainchart.io

\section{Acknowledgements}

RAIB is supported by a British Academy Postdoctoral fellowship and Autism Research Trust. JS is supported by NIMH T32MH019112-29 and K08MH120564. SRW was funded by UKRI Medical Research Council MC_UU_00002/2 and was supported by the NIHR Cambridge Biomedical Research Centre (BRC-1215-20014). ETB is supported by an NIHR Senior Investigator award. AFA-B is supported by $\mathrm{NIMH} \mathrm{K08MH120564.} \mathrm{Data} \mathrm{were} \mathrm{curated} \mathrm{and} \mathrm{analysed} \mathrm{using} \mathrm{a}$ computational facility funded by an MRC research infrastructure award (MR/M009041/1) and supported by the NIHR Cambridge Biomedical Research Centre. The views expressed are those of the authors and not necessarily those of the NIH, NHS, the NIHR or the Department of Health and Social Care. See supplementary materials for a comprehensive list of all contributing authors.

\section{Data and code availability}

All code is made available on https://github.com/ucam-department-of-psychiatry/Lifespan and summary statistics are provided in the supplementary tables and through www.brainchart.io. Sharing or re-sharing MRI scans aggregated here is through application procedures at the discretion of each contributing study individually.

\section{Contributions}

RAIB, JS, SRW, ETB and AFA-B designed the study, conducted analyses, wrote and edited the manuscript. All other authors consulted on the study design, collected, processed and contributed data and edited the manuscript. JV and KA additionally contributed to the data analysis.

\section{Disclosures}

ETB serves on the scientific advisory board of Sosei Heptares and as a consultant for GlaxoSmithKline. The other authors have no conflicts of interest to disclose. The research was reviewed by the Cambridge Psychology Research Ethics Committee (PRE.2020.104) and The 
Children's Hospital of Philadelphia's Institutional Review Board (IRB 20-017874) and deemed to not require IRB or PRE oversight.

\section{References}

1. Cole, T. J. The development of growth references and growth charts. Ann. Hum. Biol. 39, 382-394 (2012).

2. Stasinopoulos, D. \& Rigby, R. Generalized Additive Models for Location Scale and Shape (GAMLSS) in R. Journal of Statistical Software, Articles 23, 1-46 (2007).

3. Gilmore, J. H., Knickmeyer, R. C. \& Gao, W. Imaging structural and functional brain development in early childhood. Nat. Rev. Neurosci. 19, 123-137 (2018).

4. Paus, T., Keshavan, M. \& Giedd, J. N. Why do many psychiatric disorders emerge during adolescence? Nat. Rev. Neurosci. 9, 947-957 (2008).

5. Vigo, D., Thornicroft, G. \& Atun, R. Estimating the true global burden of mental illness. Lancet Psychiatry 3, 171-178 (2016).

6. Volpe, J. J. Brain injury in premature infants: a complex amalgam of destructive and developmental disturbances. Lancet Neurol. 8, 110-124 (2009).

7. Nosarti, C. et al. Preterm birth and psychiatric disorders in young adult life. Arch. Gen. Psychiatry 69, E1-8 (2012).

8. Wheater, E. N. W. et al. Birth weight is associated with brain tissue volumes seven decades later, but not with age-associated changes to brain structure. bioRxiv 2020.08.27.270033 (2020) doi:10.1101/2020.08.27.270033.

9. Jack, C. R., Jr et al. Tracking pathophysiological processes in Alzheimer's disease: an updated hypothetical model of dynamic biomarkers. Lancet Neurol. 12, 207-216 (2013).

10. Heuer, K. \& Toro, R. Role of mechanical morphogenesis in the development and evolution of the neocortex. Phys. Life Rev. 31, 233-239 (2019).

11. Werling, D. M. et al. Whole-Genome and RNA Sequencing Reveal Variation and 
Transcriptomic Coordination in the Developing Human Prefrontal Cortex. Cell Rep. 31, 107489 (2020).

12. Gandal, M. J., Leppa, V., Won, H., Parikshak, N. N. \& Geschwind, D. H. The road to precision psychiatry: translating genetics into disease mechanisms. Nat. Neurosci. 19, 1397-1407 (2016).

13. Opel, N. et al. Cross-Disorder Analysis of Brain Structural Abnormalities in Six Major Psychiatric Disorders: A Secondary Analysis of Mega- and Meta-analytical Findings From the ENIGMA Consortium. Biol. Psychiatry 88, 678-686 (2020).

14. Peterson, M. R. et al. Normal childhood brain growth and a universal sex and anthropomorphic relationship to cerebrospinal fluid. J. Neurosurg. Pediatr. 1-11 (2021).

15. Bycroft, C. et al. The UK Biobank resource with deep phenotyping and genomic data. Nature 562, 203-209 (2018).

16. Casey, B. J. et al. The Adolescent Brain Cognitive Development (ABCD) study: Imaging acquisition across 21 sites. Dev. Cogn. Neurosci. 32, 43-54 (2018).

17. Zöllei, L., Iglesias, J. E., Ou, Y., Grant, P. E. \& Fischl, B. Infant FreeSurfer: An automated segmentation and surface extraction pipeline for T1-weighted neuroimaging data of infants 0-2 years. Neuroimage 218, 116946 (2020).

18. Kim, H. et al. NEOCIVET: Towards accurate morphometry of neonatal gyrification and clinical applications in preterm newborns. Neuroimage 138, 28-42 (2016).

19. Borghi, E. et al. Construction of the World Health Organization child growth standards: selection of methods for attained growth curves. Stat. Med. 25, 247-265 (2006).

20. Pomponio, R. et al. Harmonization of large MRI datasets for the analysis of brain imaging patterns throughout the lifespan. Neuroimage 208, 116450 (2020).

21. Knickmeyer, R. C. et al. A structural MRI study of human brain development from birth to 2 years. J. Neurosci. 28, 12176-12182 (2008).

22. Gilmore, J. H. et al. Individual Variation of Human Cortical Structure Is Established in the 
First Year of Life. Biol Psychiatry Cogn Neurosci Neuroimaging 5, 971-980 (2020).

23. Courchesne, E. et al. Normal brain development and aging: quantitative analysis at in vivo MR imaging in healthy volunteers. Radiology 216, 672-682 (2000).

24. Narvacan, K., Treit, S., Camicioli, R., Martin, W. \& Beaulieu, C. Evolution of deep gray matter volume across the human lifespan. Hum. Brain Mapp. 38, 3771-3790 (2017).

25. Desikan, R. S. et al. An automated labeling system for subdividing the human cerebral cortex on MRI scans into gyral based regions of interest. Neuroimage 31, 968-980 (2006).

26. Vasung, L. et al. Association between Quantitative MR Markers of Cortical Evolving Organization and Gene Expression during Human Prenatal Brain Development. Cereb. Cortex 31, 3610-3621 (2021).

27. Fjell, A. M. et al. Development and aging of cortical thickness correspond to genetic organization patterns. Proc. Natl. Acad. Sci. U. S. A. 112, 15462-15467 (2015).

28. Sydnor, V. J. et al. Neurodevelopment of the association cortices: Patterns, mechanisms, and implications for psychopathology. Neuron 109, 2820-2846 (2021).

29. Tanner, J. M. Growth at adolescence, 2nd ed. 2, (1962).

30. Bozzola, M. \& Meazza, C. Growth Velocity Curves: What They Are and How to Use Them. Handbook of Growth and Growth Monitoring in Health and Disease 2999-3011 (2012) doi:10.1007/978-1-4419-1795-9_180.

31. Tanner, J. M., Whitehouse, R. H. \& Takaishi, M. Standards from birth to maturity for height, weight, height velocity, and weight velocity: British children, 1965. I. Arch. Dis. Child. 41, 454-471 (1966).

32. Holland, D. et al. Structural growth trajectories and rates of change in the first 3 months of infant brain development. JAMA Neurol. 71, 1266-1274 (2014).

33. Huttenlocher, P. R. \& Dabholkar, A. S. Regional differences in synaptogenesis in human cerebral cortex. J. Comp. Neurol. 387, 167-178 (1997).

34. Petanjek, Z. et al. Extraordinary neoteny of synaptic spines in the human prefrontal cortex. 
Proc. Natl. Acad. Sci. U. S. A. 108, 13281-13286 (2011).

35. Miller, D. J. et al. Prolonged myelination in human neocortical evolution. Proc. Natl. Acad. Sci. U. S. A. 109, 16480-16485 (2012).

36. Tau, G. Z. \& Peterson, B. S. Normal development of brain circuits. Neuropsychopharmacology 35, 147-168 (2010).

37. Mountcastle, V. B. The columnar organization of the neocortex. Brain 120, 4, 701-722 (1997).

38. Blüml, S. et al. Metabolic maturation of the human brain from birth through adolescence: insights from in vivo magnetic resonance spectroscopy. Cereb. Cortex 23, 2944-2955 (2013).

39. Kuzawa, C. W. et al. Metabolic costs and evolutionary implications of human brain development. Proc. Natl. Acad. Sci. U. S. A. 111, 13010-13015 (2014).

40. WHO MULTICENTRE GROWTH REFERENCE STUDY GROUP \& Onis, M. WHO Motor Development Study: Windows of achievement for six gross motor development milestones. Acta Paediatr. 95, 86-95 (2007).

41. Solmi, M. et al. Age at onset of mental disorders worldwide: large-scale meta-analysis of 192 epidemiological studies. Mol. Psychiatry 1-15 (2021).

42. Kang, H. J. et al. Spatio-temporal transcriptome of the human brain. Nature 478, 483-489 (2011).

43. Sorva, R., Lankinen, S., Tolppanen, E. M. \& Perheentupa, J. Variation of growth in height and weight of children. II. After infancy. Acta Paediatr. Scand. 79, 498-506 (1990).

44. Elliott, L. T. et al. Genome-wide association studies of brain imaging phenotypes in UK Biobank. Nat. Neurosci. 562, 210-216 (2018).

45. Marquand, A. F., Rezek, I., Buitelaar, J. K. \& Beckmann, C. F. Understanding Heterogeneity in Clinical Cohorts Using Normative Models: Beyond Case-Control Studies. Biol. Psychiatry 80, 552-561 (2016). 
46. Sharma, E. et al. Consortium on Vulnerability to Externalizing Disorders and Addictions (cVEDA): A developmental cohort study protocol. BMC Psychiatry 20, 2 (2020).

47. Liu, S. et al. Chinese Color Nest Project : An accelerated longitudinal brain-mind cohort.

Dev. Cogn. Neurosci. 52, 101020 (2021).

48. Shansky, R. M. \& Murphy, A. Z. Considering sex as a biological variable will require a global shift in science culture. Nat. Neurosci. 24, 457-464 (2021).

\section{Online methods: Brain charts for the human lifespan}

To accurately and comprehensively establish standardised brain reference charts across the lifespan, it is crucial to leverage multiple independent and diverse datasets, especially those spanning prenatal and early postnatal life. Here we sought to chart normative brain development and ageing across the largest age-span and largest aggregated neuroimaging dataset to date using a robust and scalable methodological framework ${ }^{1,2}$. We leveraged these normative reference charts in clinical cohorts to generate individualised assessments of age-relative centiles. These centiles were then leveraged to investigate cross-diagnostic and longitudinal atypicalities of brain morphology across the lifespan. We used generalised additive models for location, scale and shape (GAMLSS) ${ }^{1}$ to estimate cross-sectional normative age-related trends from 100 studies (see supplementary tables [ST] 1.1-1.7 for full demographic information and supplementary information [SI] 19 for dataset descriptions). The GAMLSS approach allows not only modelling of age-related changes in brain phenotypes but also age related-changes in the variability, in the form of both linear and nonlinear changes over time, thereby overcoming potential limitations of conventional additive models that only allow additive means to be modelled $^{1}$. In addition, site-specific offsets (mean and variance) for each brain phenotype are also modelled. These modelling criteria are particularly important in the context of establishing growth references as recommended by the World Health Organisation ${ }^{2}$, as it is reasonable to assume the distribution of higher order moments (e.g., variance) changes with age, sex, site/study and pre-processing pipeline-especially given the impossibility of fully comprehensive longitudinal data for individuals spanning the $\sim 100$ year age range. Furthermore, recent studies suggest that changes in across-individual variability might intersect with vulnerability for developing a mental health condition ${ }^{3}$. The use of data spanning the entire age range is also critical, as estimation from partial age-windows can lead to biased estimations when extrapolated to the whole lifespan. In summary, using a sex-stratified approach ${ }^{2}$, age, preprocessing pipeline and study were each included in the GAMLSS model estimation of first order $(\mu)$ and second order $(\sigma)$ distribution parameters of a generalised gamma distribution using fractional polynomials to model nonlinear trends. See Supplementary Information [SI] for more details regarding GAMLSS model specification and estimation (SI1), image quality control (SI2), model stability and robustness (SI34), phenotypic validation against non-imaging metrics (SI3 \& SI5.2), inter-study harmonisation (SI5) and assessment of cohort effects (SI6). 
In general, the GAMLSS framework can be specified in the following way:

$$
\begin{gathered}
Y \sim \mathcal{F}(\mu, \sigma, v, \tau) \\
g_{\mu}(\mu)=X_{\mu} \beta_{\mu}+Z_{\mu} \gamma_{\mu}+\sum_{i} s_{\mu, i}\left(x_{i}\right) \\
g_{\sigma}(\sigma)=X_{\sigma} \beta_{\sigma}+Z_{\sigma} \gamma_{\sigma}+\sum_{i} s_{\sigma, i}\left(x_{i}\right) \\
g_{v}(v)=X_{v} \beta_{v}+Z_{v} \gamma_{v}+\sum_{i} s_{v, i}\left(x_{i}\right) \\
g_{\tau}(\tau)=X_{\tau} \beta_{\tau}+Z_{\tau} \gamma_{\tau}+\sum_{i} s_{\tau, i}\left(x_{i}\right)
\end{gathered}
$$

Here, the outcome vector, $Y$, follows a probability distribution $D$ parameterised by up to four parameters, $(\mu, \sigma, v, \tau)$. The four parameters, depending on the parameterisation of the probability density function, may correspond to the mean, variance, skewness, and kurtosis (i.e., the first four moments); however, for many distributions there is not a direct one-to-one correspondence. Each component is linked to a linear equation through a link-function, $g$.(), and each component equation may include three types of terms: fixed effects, $\beta$. (with design matrix, $X$.); random-effects, $\gamma$. (with design matrix, Z.); and non-parametric smoothing functions, $s_{, i}$ applied to the $i^{\text {th }}$ covariate. The nature of the outcome distribution determines the appropriate link-functions and which components are used. In principle any outcome distribution can be used, from well-behaved continuous and discrete outcomes, through to mixtures and truncations.

Within this paper we consider fractional polynomials as a flexible, yet limited in complexity, approach to modelling age-related changes. Although non-parametric smoothers are more flexible, they can become unstable and infeasible, especially in the presence of random-effects. Hence, the fractional polynomials enter the model within the X.terms, with associated coefficients in $\beta$. The GAMLSS framework includes the ability to estimate the most appropriate powers within the iterative fitting algorithm, searching across the standard set of powers, $p \in$ $\{-2,-1,-0.5,0,0.5,1,2,3\}$, where the design matrix includes the covariate (in our setting, age) raised to the power, namely, $x^{p}$. Fractional polynomials naturally extend to higher-orders, for example a second-order fractional polynomial of the form, $x^{p_{1}}+x^{p_{2}}$.

There are several options for including random-effects within the GAMLSS framework depending on the desired covariance structures. We consider the simplest case, including a factor-level (or group-level) random-intercept, where the observations are grouped by the study covariate. The random-effects are drawn from a normal distribution with zero mean and variance to be estimated, $\gamma . \sim N\left(0, \delta^{2}\right)$. The ability to include random-effects is fundamental to accounting for codependence between observations. It is therefore possible to take advantage of the flexibility of "standard" GAMLSS, as typically used to develop growth charts ${ }^{2,4,5}$, while accounting for codependence between observations using random-effects. The typical applications of GAMLSS assume independent and identically distributed outcomes; however within our context it is 
essential to account for within-study covariance implying the observations are no longer independent.

This model allowed us to leverage the aggregated life-spanning neuroimaging dataset to derive developmental milestones (i.e., peaks of trajectories) and compare them to existing literature. These sex-stratified models incorporated variation in study and processing pipeline to allow computation of standardised reference charts. The cerebrum tissue classes from 100 studies (Fig. 1A, ST1.1-1.7, SI18) showed clear, predominantly age-related trends, even prior to any modelling (Fig. 1B). Yet, marked heterogeneity of growth curves for individual studies (www.brainchart.io) reinforces the importance of using the full aggregated dataset to achieve representative norms not biased by individual studies. The validity of the models is supported by high stability under cross-validation and bootstrap resampling (SI3). Comparing these models to multiple non-MRI metrics of brain size demonstrated high correspondence across the lifespan (SI3). Peaks were determined based on the GAMLSS model output ( $50^{\text {th }}$ centile) for each of the tissue classes and TCV, for both total tissue volumes (Fig. 1B) and rates of change or growth ("velocity"; Fig. 1E). Diagnostic age ranges from previous literature ${ }^{6,7}$ were plotted (blue boxes in Fig. 3) to compare with empirical age ranges of patients with a given diagnosis across the aggregated neuroimaging dataset (black boxes in Fig. 3). Note that age of diagnosis is significantly later than age of symptom onset for many disorders ${ }^{6}$. Developmental milestones were re-plotted based on published work for brain resting metabolic rate $(\mathrm{RMR})^{8}$, from its minimum in infancy to its maximum in early childhood; anthropometric variables (height and weight), which reach a first peak in velocity during infancy and a second peak in velocity in adolescence ${ }^{9}$; and typical acquisition of the six gross motor capabilities ${ }^{4}$. Pubertal age ranges were taken from reported typical age ranges ${ }^{10,11}$.

Furthermore, these neuroimaging-derived brain reference charts also enabled each individual to be quantified relative to a statistical distribution defined at the reference level for any point during the lifespan ${ }^{12,13}$. Individual centile scores were obtained relative to the reference curves, conceptually similar to traditional anthropometric growth charts. These normative scores represent a novel set of population and age standardised clinical phenotypes, providing the capacity for cross-phenotype, cross-study and cross-disorder comparison. A single summary deviation metric for each individual was also generated. Main group effects were analysed with a bootstrapped (500 bootstraps) non-parametric generalisation of Welch's one-way ANOVA. Pairwise, sex stratified, post-hoc comparisons were conducted using non-parametric Monte-Carlo permutation tests $(10,000$ permutations) and thresholded at a Benjamini-Hochberg false discovery rate (FDR) of $q<0.05$.

To utilise the centiles in a diagnostically meaningful or predictive way, they need to be stable across multiple measuring points. To assess this intra-individual stability we calculated the subject specific interquartile range (IQR) of centiles across timepoints for the datasets that included longitudinal scans ( $n=9,306,41$ unique studies). Exploratory longitudinal clinical analyses were restricted to clinical groups that had at least 50 subjects with longitudinal data to allow for robust group-wise estimates of longitudinal variability. In addition, there was a small subset of individuals with documented pathological progression across longitudinal scans, for instance from high risk 
status to formal diagnosis. Here, we would expect an associated change in centile measurement. To test this hypothesis we assessed whether these individuals showed differences in centile variability (as assessed with IQR), and their approximate direction of change.

Finally, we provide an interactive tool (www.brainchart.io) and have made our code and models openly available (https://github.com/ucam-department-of-psychiatry/Lifespan). The tool not only allows the user to visualise the underlying datasets' demographics and reported reference charts in a much more detailed fashion than static images allow, it also provides the opportunity for interactive exploration of differences in centile scores across many clinical groups that is beyond the present manuscript. Perhaps most significantly, it includes an out-of-sample estimator of model parameters for novel data that enables the user to compute percentile scores for their own datasets without the computational or data-sharing hurdles involved in adding that data to the reference chart. All modelling included extensive validation, sensitivity analyses and multi-modal validation against existing growth chart references.

Though already based on the largest and most comprehensive neuroimaging dataset to date and supporting analyses of out-of-sample data, the underlying reference charts will also be updated as additional data is made available.

\section{References}

1. Stasinopoulos, D. \& Rigby, R. Generalized Additive Models for Location Scale and Shape (GAMLSS) in R. Journal of Statistical Software, Articles 23, 1-46 (2007).

2. Borghi, E. et al. Construction of the World Health Organization child growth standards: selection of methods for attained growth curves. Stat. Med. 25, 247-265 (2006).

3. Wierenga, L. M. et al. Greater male than female variability in regional brain structure across the lifespan. Hum. Brain Mapp. (2020) doi:10.1002/hbm.25204.

4. WHO MULTICENTRE GROWTH REFERENCE STUDY GROUP \& Onis, M. WHO Motor Development Study: Windows of achievement for six gross motor development milestones. Acta Paediatr. 95, 86-95 (2007).

5. Heude, B. et al. A big-data approach to producing descriptive anthropometric references: a feasibility and validation study of paediatric growth charts. Lancet Digit Health 1, e413e423 (2019). 
6. Solmi, M. et al. Age at onset of mental disorders worldwide: large-scale meta-analysis of 192 epidemiological studies. Mol. Psychiatry 1-15 (2021).

7. Erkkinen, M. G., Kim, M.-O. \& Geschwind, M. D. Clinical Neurology and Epidemiology of the Major Neurodegenerative Diseases. Cold Spring Harb. Perspect. Biol. 10, (2018).

8. Kuzawa, C. W. et al. Metabolic costs and evolutionary implications of human brain development. Proc. Natl. Acad. Sci. U. S. A. 111, 13010-13015 (2014).

9. Bozzola, M. \& Meazza, C. Growth Velocity Curves: What They Are and How to Use Them. Handbook of Growth and Growth Monitoring in Health and Disease 2999-3011 (2012) doi:10.1007/978-1-4419-1795-9_180.

10. Tanner, J. M., Whitehouse, R. H. \& Takaishi, M. Standards from birth to maturity for height, weight, height velocity, and weight velocity: British children, 1965. I. Arch. Dis. Child. 41, 454-471 (1966).

11. Tanner, J. M. Growth at adolescence, 2nd ed. 2, (1962).

12. Marquand, A. F., Rezek, I., Buitelaar, J. K. \& Beckmann, C. F. Understanding Heterogeneity in Clinical Cohorts Using Normative Models: Beyond Case-Control Studies. Biol. Psychiatry 80, 552-561 (2016).

13. Bethlehem, R. A. I. et al. A normative modelling approach reveals age-atypical cortical thickness in a subgroup of males with autism spectrum disorder. Commun Bio/ 3, 486 (2020). 


\section{Affiliations}

${ }^{1}$ Autism Research Centre, Department of Psychiatry, University of Cambridge, Cambridge, CB2 0SZ, UK.

${ }^{2}$ Brain Mapping Unit, Department of Psychiatry, University of Cambridge, Cambridge, CB2 OSZ, UK

${ }^{3}$ Department of Psychiatry, University of Pennsylvania, Philadelphia, PA 19104

${ }^{4}$ Department of Child and Adolescent Psychiatry and Behavioral Science, The Children's Hospital of Philadelphia, Philadelphia, PA 19104

${ }^{5}$ Department of Psychiatry, University of Cambridge, Cambridge, CB2 0SZ, UK

${ }^{6}$ MRC Biostatistics Unit, University of Cambridge, Cambridge, England

${ }^{7}$ Lifespan Informatics \& Neuroimaging Center, University of Pennsylvania, Philadelphia, PA 19104

${ }^{8}$ Department of Psychology, Yale University, New Haven, CT, USA

${ }^{9}$ Developmental Imaging, Murdoch Children's Research Institute, Melbourne, Victoria, Australia

${ }^{10}$ Department of Medicine, Monash University, Melbourne, Victoria, Australia

11 UCL Great Ormond Street Institute for Child Health, 30 Guilford St, Holborn, London WC1N 1EH

${ }^{12}$ Weill Cornell Institute of Geriatric Psychiatry, Department of Psychiatry, Weill Cornell Medicine

${ }^{13}$ Department of Pediatrics University of Toronto

${ }^{14}$ Holland Bloorview Kids Rehabilitation Hospital, Toronto, Canada

${ }^{15}$ The Clinical Hospital of Chengdu Brain Science Institute, MOE Key Lab for Neurolnformation, University of Electronic Science and Technology of China, No. 2006, Xiyuan Ave., West Hi-Tech Zone, Chengdu, 611731, China ${ }^{16}$ University of Pinar del Río "Hermanos Saiz Montes de Oca", Cuba

${ }^{17}$ MRC Cognition and Brain Sciences Unit, University of Cambridge, Cambridge UK

${ }^{18}$ Department of Psychology, School of Philosophy, Psychology and Language Sciences, University of Edinburgh, Edinburgh, United Kingdom

${ }_{19}$ University College London, Mental Health Neuroscience Research Department, Division of Psychiatry, London UK

${ }^{20}$ Department of Paediatrics, University of Melbourne, Melbourne, Victoria, Australia

${ }^{21}$ Cambridge Lifetime Asperger Syndrome Service (CLASS), Cambridgeshire and Peterborough NHS Foundation Trust, Cambridge, United Kingdom

${ }^{22}$ Centre for Addiction Medicine, National Institute of Mental Health and Neurosciences, Bengaluru, India 560029

${ }^{23}$ Department of Neurology, Max Planck Institute for Human Cognitive and Brain Sciences, Leipzig, 04103, Germany

${ }^{24}$ Department of Neuropsychiatry, Seoul National University Bundang Hospital, Seongnam, Korea

${ }^{25}$ Department of Human Genetics, South Texas Diabetes and Obesity Institute, University of Texas Rio Grande Valley

${ }^{26}$ MRC Centre for Reproductive Health, University of Edinburgh, UK

${ }^{27}$ Fetal and Neonatal Institute, Division of Neonatology, Children's Hospital Los Angeles, Department of Pediatrics, Keck School of Medicine, University of Southern California, Los Angeles, California USA

${ }^{28}$ McGill Centre for Integrative Neuroscience, Ludmer Centre for Neuroinformatics and Mental Health, Montreal Neurological Institute

${ }^{29}$ McGill University 
${ }^{30}$ Department of Brain Sciences, Imperial College London, London UK \& Care Research \& Technology Centre, UK Dementia Research Institute

${ }^{31}$ Tri-institutional Center for Translational Research in Neuroimaging and Data Science, Georgia State University, Georgia Institute of Technology, and Emory University, Atlanta, GA, USA

${ }^{32}$ Computational Brain Anatomy (CoBrA) Laboratory, Cerebral Imaging Centre, Douglas Mental Health University Institute

${ }^{33}$ Penn Statistics in Imaging and Visualization Center, Department of Biostatistics, Epidemiology, and Informatics, Perelman School of Medicine, University of Pennsylvania, Philadelphia, PA, USA

${ }^{34}$ Normandie Univ, UNICAEN, INSERM, U1237, PhIND "Physiopathology and Imaging of Neurological Disorders", Institut Blood and Brain @ Caen-Normandie, Cyceron, 14000 Caen, France

${ }^{35}$ Singapore Institute for Clinical Sciences, Agency for Science, Technology and Research, Singapore

${ }^{36}$ Department of Obstetrics and Gynaecology, Yong Loo Lin School of Medicine, National University of Singapore, Singapore

${ }^{37}$ Centre for Medical Image Computing (CMIC), University College London

${ }^{38}$ Dementia Research Centre (DRC), University College London

${ }^{39}$ Department of Psychiatry, Trinity College, Dublin, Ireland

${ }^{40}$ Cerebral Imaging Centre, Douglas Mental Health University Institute, Verdun, Canada

${ }^{41}$ Undergraduate program in Neuroscience, McGill University, Montreal, Canada

${ }^{42}$ Department of Neuroscience, University of California, San Diego, San Diego, CA 92093, USA

${ }_{43}^{43}$ Autism Center of Excellence, University of California, San Diego, San Diego, CA 92037, USA

44 Institute of Neurodegenerative Disorders, CNRS UMR5293, University of Bordeaux

${ }^{45}$ Melbourne Neuropsychiatry Centre, University of Melbourne, Melbourne, Australia

${ }^{46}$ The Hospital for Sick Children, Toronto, Canada

${ }^{47}$ Department of Psychiatry, School of Medicine, Pontificia Universidad Católica de Chile, Diagonal Paraguay 362, Santiago 8330077, Chile

${ }^{48}$ Department of Psychosis Studies, Institute of Psychiatry, Psychology and Neuroscience, King's College London, De Crespigny Park, London SE5 8AF, UK

${ }^{49}$ Child and Adolescent Psychiatry Department, Robert Debré University Hospital, AP-HP, F-75019, Paris France

${ }^{50}$ Human Genetics and Cognitive Functions , Institut Pasteur, F-75015, Paris France

${ }^{51}$ Social, Genetic and Developmental Psychiatry Centre, Institute of Psychiatry, Psychology \& Neuroscience, King's College London, London, United Kingdom

${ }^{52}$ Cerebral Imaging Centre, Douglas Mental Health University Institute, Montreal, QC, Canada

${ }^{53}$ Department of Psychiatry, McGill University, Montreal, QC, Canada

${ }^{54}$ Department of Psychiatry, Brigham and Women's Hospital, Harvard Medical School, Boston, Massachusetts,

United States

${ }^{55}$ Max Planck UCL Centre for Computational Psychiatry and Ageing Research, University College London, London, UK.

${ }^{56}$ Wellcome Centre for Human Neuroimaging, University College London, London, UK

${ }^{57}$ University of Cape Town, South Africa, Cape Town, South Africa

${ }^{58}$ Center for Neuroimaging, Cognition \& Genomics (NICOG), School of Psychology, National University of Ireland Galway, Galway, Ireland

${ }^{59}$ Weil Family Brain and Mind Research Institute, Department of Psychiatry, Weill Cornell Medicine

${ }^{60}$ Centre for the Developing Brain, King's College London, London, UK

${ }^{61}$ Evelina London Children's Hospital

${ }^{62}$ Institute of Child Development, Department of Pediatrics, Masonic Institute for the Developing Brain, University of Minnesota, Minneapolis, MN, United States

${ }^{63}$ Department of Psychiatry, Center for Behavior Genetics of Aging, University of California, San Diego, La Jolla, CA ${ }^{64}$ Desert-Pacific Mental Illness Research Education and Clinical Center, VA San Diego Healthcare, San Diego, CA, USA

${ }^{65}$ Department of Psychiatry, University of California San Diego, Los Angeles, CA, USA

${ }^{66}$ Department of Psychiatry, University of Cambridge, and Wellcome Trust MRC Institute of Metabolic Science, Cambridge Biomedical Campus, Cambridge, United Kingdom

${ }^{67}$ Cambridgeshire and Peterborough NHS Foundation Trust

${ }^{68}$ Department of Clinical, Educational and Health Psychology, University College London, London, UK 
${ }^{69}$ Department of Psychiatry, Center for Behavior Genetics of Aging, University of California, San Diego, La Jolla, CA 92093

${ }^{70}$ Cuban Center for Neuroscience, La Habana, Cuba

${ }^{71}$ Computational Radiology Laboratory, Boston Children's Hospital, Boston, MA 02115

72 Department of Child and Adolescent Psychiatry, University of California, San Diego, San Diego, CA 92093, USA

${ }^{73}$ Department of Psychiatry, University of California San Diego, San Diego, CA, USA

${ }^{74}$ Department of Psychiatry, University of North Carolina, Chapel Hill, NC, USA

${ }^{75}$ Department of Psychiatry, Boston Children's Hospital and Harvard Medical School, Boston, MA 02115

${ }^{76}$ Harvard Medical School, Boston, MA 02115

${ }^{77}$ Division of Newborn Medicine and Neuroradiology, Fetal Neonatal Neuroimaging and Developmental Science Center, Boston Children's Hospital, Harvard Medical School, Boston, MA 02115, USA

${ }^{78}$ Department of Paediatrics and Child Health, Red Cross War Memorial Children's Hospital, SA-MRC Unit on Child \& Adolescent Health, University of Cape Town, South Africa

${ }^{79}$ Neuroscience Institute, University of Cape Town, Cape Town, South Africa

${ }^{80}$ Lifespan Brain Institute, The Children's Hospital of Philadelphia, Philadelphia, PA 19104

${ }^{81}$ Lifespan Brain Institute, The Children's Hospital of Philadelphia, Philadelphia, PA 19105

${ }^{82}$ Mouse Imaging Centre, Toronto, Canada

${ }^{83}$ Clinical Memory Research Unit, Department of Clinical Sciences Malmö, Lund University, Malmö, Sweden

${ }^{84}$ Memory Clinic, Skåne University Hospital, Malmö, Sweden

${ }^{85}$ Department of Neurology, Icahn School of Medicine at Mount Sinai, New York, NY 10029, USA

${ }^{86}$ Athinoula A. Martinos Center for Biomedical Imaging, Department of Radiology, Massachusetts General Hospital, Harvard Medical School, Boston, MA 02129, USA

${ }^{87}$ Department of Psychiatry and Psychotherapy, Charite University Hospital Berlin, Berlin, Germany

${ }^{88}$ Department of Psychiatry, University of Cambridge, Cambridge, UK

${ }^{89}$ Department of Neuropsychology, Max Planck Institute for Human Cognitive and Brain Sciences, Leipzig, Germany

90 Université de Paris, Paris, France

${ }^{91}$ Department of Psychiatry, University of Cape Town, Cape Town, South Africa.

${ }^{92}$ Departments of Psychiatry and Integrative Medicine, NIMHANS, Bengaluru, India

${ }_{93}$ Departments of Psychology and Psychiatry, Yale University, New Haven, CT, USA

${ }^{94}$ Radiology Research, Children's Hospital of Philadelphia, Philadelphia, United States

${ }_{95}^{95}$ The Department of Radiology, Perelman School of Medicine, University of Pennsylvania, Philadelphia, United States

${ }^{96}$ Division of Newborn Medicine, Fetal Neonatal Neuroimaging and Developmental Science Center, Boston

Children's Hospital, Harvard Medical School, Boston, MA 02115, USA

${ }^{97}$ Boston Children's Hospital, Boston, MA 02115

${ }^{98}$ Department of Psychiatry and Mental Health, Clinical Neuroscience Institute, University of Cape Town

${ }^{99}$ Department of Radiology, Mayo Clinic, Rochester, MN 55905, USA

${ }^{100}$ Department of Psychiatry, Universidade Federal de São Paulo

${ }^{101}$ National Institute of Developmental Psychiatry, CNPq

102 Institute of Science and Technology for Brain-Inspired Intelligence, Fudan University, Shanghai, 200433, China

${ }^{103}$ Key Laboratory of Computational Neuroscience and BrainInspired Intelligence (Fudan University), Ministry of Education, Shanghai, China

${ }^{104}$ Centre for Population Neuroscience and Precision Medicine (PONS), Institute of Psychiatry, Psychology and Neuroscience, SGDP Centre, King's College London, London SE5 8AF, UK

${ }^{105}$ Harvard Aging Brain Study, Department of Neurology, Massachusetts General Hospital, Boston, MA 02114

${ }^{106}$ Center for Alzheimer Research and Treatment, Department of Neurology, Brigham and Women's Hospital,

Boston, MA 02115

${ }^{107}$ Department of Radiology, Massachusetts General Hospital, Boston, MA

${ }^{108}$ Cambridgeshire and Peterborough NHS Foundation Trust, Huntingdon, United Kingdom

${ }^{109}$ Department of Neurology, Mayo Clinic, Rochester, MN, USA

110 Department of Radiology, Mayo Clinic, Rochester, MN, USA

${ }^{111}$ Department of Psychiatry Division of Neurosciences, University Medical Center, Utrecht, The Netherlands

112 Department of Psychiatry, Icahn School of Medicine, Mount Sinai, New York, USA 
${ }^{113}$ Department of Clinical Medicine, Department of Psychiatry and Turku Brain and Mind Center, FinnBrain Birth Cohort Study, University of Turku and Turku University Hospital, Turku, Finland

${ }^{114}$ Centre for Population Health Research, Turku University Hospital and University of Turku, Turku, Finland

115 Institute of Development, Aging and Cancer, Tohoku University, Seiryocho, Aobaku, Sendai 980-8575, Japan

${ }^{116}$ Queen's University, Departments of Psychology and Psychiatry, Centre for Neuroscience Studies, Kingston,

Ontario, Canada

117 Institute of Neuroscience and Physiology, University of Gothenburg, Gothenburg, Sweden

${ }^{118}$ Department of Brain and Cognitive Sciences, Seoul National University College of Natural Sciences, Seoul,

Republic of Korea

119 Department of Neuropsychiatry, Seoul National University Bundang Hospital, Seongnam, Republic of Korea

${ }^{120}$ Department of Psychiatry, Seoul National University College of Medicine, Seoul, Republic of Korea

${ }^{121}$ Department of Brain and Cognitive Science, Seoul National University College of Natural Sciences

${ }^{122}$ Section on Developmental Neurogenomics, Human Genetics Branch, National Institute of Mental Health,

Bethesda, MD, USA

${ }^{123}$ Department of Brain \& Cognitive Sciences, Seoul National University College of Natural Sciences, Seoul, Korea

${ }^{124}$ Department of Medical Biophysics, University of Toronto, Toronto, ON, Canada

${ }^{125}$ Mouse Imaging Centre, The Hospital for Sick Children, Toronto, ON, Canada

${ }^{126}$ Wellcome Centre for Integrative Neuroimaging, FMRIB, Nuffield Department of Clinical Neuroscience, University of Oxford, Oxford, UK

${ }^{127}$ Montreal Neurological Institute, McGill University, Montreal, Canada

${ }^{128}$ The Clinical Hospital of Chengdu Brain Science Institute, University of Electronic Science and Technology of

China, Chengdu 611731, China

${ }^{129}$ Department of Psychiatry and Brain and Mind Research Institute, Weill Cornell Medicine

${ }^{130}$ Laboratory for Autism and Neurodevelopmental Disorders, Center for Neuroscience and Cognitive Systems

@UniTn, Istituto Italiano di Tecnologia, Rovereto, Italy

${ }^{131}$ School of Biomedical Engineering \& Brain and Mind Centre, The University of Sydney, Sydney, NSW, Australia

132 Department of Psychology, University of Texas, Austin, Texas 78712, USA

${ }^{133}$ Department of Psychiatry and Neuropsychology, School of Mental Health and Neuroscience, EURON, Maastricht

University Medical Centre, PO Box 616, 6200 MD, Maastricht, the Netherlands; Institute for Mental Health Care

Eindhoven (GGzE), Eindhoven, the Netherlands.

${ }^{134}$ McConnell Brain Imaging Centre, Montreal Neurological Institute, McGill University, Montreal, QC H3A 2B4, Canada

${ }^{135}$ Bordeaux University Hospital

${ }^{136}$ Professor, Department of Psychosis Studies, Institute of Psychiatry, Psychology and Neuroscience, King's College London, UK

${ }^{137}$ Ludmer Centre for Neuroinformatics and Mental Health, Douglas Mental Health University Institute, McGill

University, Montreal, Quebec, Canada; Singapore Institute for Clinical Sciences, Singapore

${ }^{138}$ Department of Computer Science and Technology, University of Cambridge, Cambridge CB3 OFD, United

Kingdom

139 Department of Psychiatry, University of Cambridge, Cambridge CB2 0SZ, United Kingdom

${ }_{140}$ The Alan Turing Institute, London, NW1 2DB

${ }^{141}$ Department of Psychology, School of Business, National College of Ireland, Dublin, Ireland

${ }^{142}$ School of Psychology \& Center for Neuroimaging and Cognitive Genomics, National University of Ireland Galway,

Galway, Ireland

${ }^{143}$ Department of Psychiatry, Trinity College Dublin, Dublin, Ireland

${ }^{144}$ Department of Psychiatry, School of Medicine, Oregon Health and Science University, Portland, United States

${ }^{145}$ Center for Sleep and Cognition, Yong Loo Lin School of Medicine, National University of Singapore, Singapore

${ }^{146}$ Department of Pediatrics, Washington University in St. Louis, St. Louis, Missouri, United States

${ }^{147}$ Alzheimer Center Amsterdam, Department of Neurology, Amsterdam Neuroscience, Vrije Universiteit Amsterdam, Amsterdam UMC, Amsterdam, The Netherlands

148 Lund University, Clinical Memory Research Unit, Lund, Sweden

${ }^{149}$ Robarts Research Institute \& The Brain and Mind Institute, University of Western Ontario,London, Ontario,Canada.

150 Department of Psychiatry, Federal University of Sao Poalo (UNIFESP)

${ }^{151}$ National Institute of Developmental Psychiatry for Children and Adolescents (INPD), Brazil. 
152 Melbourne Neuropsychiatry Centre, Department of Psychiatry, The University of Melbourne and Melbourne Health, Carlton South, Victoria, Australia

${ }^{153}$ Melbourne School of Engineering, The University of Melbourne, Parkville, Victoria, Australia

${ }^{154}$ Florey Institute of Neuroscience and Mental Health, Parkville, VIC, Australia

${ }^{155}$ Department of Psychiatry, Schulich School of Medicine and Dentistry, Western University, London, ON, Canada

${ }^{156}$ Department of Psychiatry, Faculty of Medicine and Centre Hospitalier Universitaire Sainte-Justine, University of Montreal, Montreal, Quebec, Canada

${ }^{157}$ Departments of Psychiatry and Psychology, University of Toronto, Toronto, ON, Canada.

${ }^{158}$ Departments of Physiology and Nutritional Sciences, University of Toronto, Toronto, Canada

${ }^{159}$ Cuban Neuroscience Center, Havana, Cuba

${ }^{160}$ Department of Psychiatry, Faculty of Medicine, McGill University, Montreal, Qc, H3A 1Y2, Canada

161 Douglas Mental Health University Institute, Montreal, Qc, H4H 1R3, Canada.

${ }^{162}$ Autism Center of Excellence, Department of Neurosciences, University of California, San Diego La Jolla, CA, USA

${ }_{163}$ School of Psychology, Southwest University, Chongqing 400715, P.R. China

${ }^{164}$ Department of Biomedical Engineering, The N.1 Institute for Health, National University of Singapore.

${ }^{165}$ Department of Clinical Neurosciences, University of Cambridge, Cambridge UK

${ }^{166}$ Department of Neurology, Harvard Medical School

${ }^{167}$ Department of Neurology, Boston Children's Hospital, Boston, MA 02115

168 Instituto de Biomedicina de Sevilla (IBiS) HUVR/CSIC/Universidad de Sevilla, Dpto. de Fisiología Médica y

Biofísica, Spain

169 Department of Psychology, Neuroscience Institute, University of Chicago

${ }^{170}$ Department of Paediatrics and Wellcome-MRC Cambridge Stem Cell Institute, University of Cambridge, Hills

Road, Cambridge, UK

171 Department of Psychiatry, Universidade Federal do Rio Grande do Sul (UFRGS)

172 National Institute of Developmental Psychiatry (INPD)

173 Otto Hahn Group Cognitive Neurogenetics, Max Planck Institute for Human Cognitive and Brain Sciences, Leipzig,

Germany

${ }^{174}$ Institute of Neuroscience and Medicine (INM-7: Brain and Behaviour), Research Centre Juelich, Juelich, Germany

${ }^{175}$ Athinoula A. Martinos Center for Biomedical Imaging, Department of Radiology, Massachusetts General Hospital,

Charlestown, MA 02129, USA

${ }^{176}$ Centre for Population Neuroscience and Stratified Medicine (PONS), SGDP Centre, loPPN, KCL, UK

177 PONS-Centre, Dept of Psychiatry and Psychotherapy, Campus Charite Mitte, Humboldt University, Berlin,

Germany

${ }_{178}$ PONS Centre, Institute for Science and Technology of Brain-inspired Intelligence (ISTBI), Fudan University,

Shanghai, China

179 Wallenberg Centre for Molecular and Translational Medicine, University of Gothenburg, Gothenburg, Sweden

${ }^{180}$ Department of Psychiatry and Neurochemistry, University of Gothenburg, Sweden

${ }^{181}$ Dementia Research Centre, Queen's Square Institute of Neurology, University College London, UK

${ }^{182}$ Center for Biomedical Image Computing and Analytics, Department of Radiology, Perelman School of Medicine,

University of Pennsylvania, Philadelphia, PA, USA

${ }^{183}$ Departments of Neurology, Pediatrics, and Radiology, Washington University School of Medicine, St. Louis, United States

${ }^{184}$ SA MRC Unit on Risk \& Resilience in Mental Disorders, Dept of Psychiatry and Neuroscience Institute, University

of Cape Town, Cape Town, South Africa

${ }^{185}$ Division of Psychiatry, Centre for Clinical Brain Sciences, University of Edinburgh, UK

${ }^{186}$ Department of Neuroscience, Institut Pasteur, Paris, France

${ }^{187}$ Center for Research and Interdisciplinarity (CRI), Université Paris Descartes, Paris, France

${ }^{188}$ Department of Psychology, University of Cambridge, Cambridge, UK

189 Wu Tsai Institute, Yale University, New Haven, CT, USA

190 Department of Clinical Medicine, Department of Psychiatry, FinnBrain Birth Cohort Study, University of Turku,

Turku, Finland

191 Department of Clinical Medicine, University of Turku, Turku Finland

192 Turku Collegium for Science, Medicine and Technology, University of Turku, Turku, Finland 
193 Univ. Bordeaux, Inserm, Bordeaux Population Health Research Center, U1219, CHU Bordeaux, F-33000

Bordeaux, France

${ }^{194}$ Department of Anesthesia, Faculty of Medicine, McGill University, Montreal, Qc, H3A 1G1, Canada

${ }^{195}$ Faculty of Dentistry, McGill University, Montreal, Qc, H3A 1G1, Canada

${ }^{196}$ Alan Edwards Centre for Research on Pain (AECRP), McGill University, Montreal, Qc, H3A 1G1, Canada

197 Joint China-Cuba Lab,University of Electronic Science and Technology, Chengdu China/Cuban Center for Neuroscience, La Habana, Cuba

198 University of Electronic Science and Technology of China/Cuban Center for Neuroscience

199 Institute for Neuroscience and Medicine 7, Forschungszentrum Juelich; Max Planck Institute for Human Cognitive and Brain Sciences

${ }^{200}$ Department of Psychiatry \& Neurosychology, Maastricht University, Maastricht, The Netherlands

${ }^{201}$ Department of Biostatistics, Vanderbilt University, Nashville, Tennessee, USA

${ }^{202}$ Department of Biostatistics, Vanderbilt University Medical Center, Nashville, Tennessee, USA

${ }^{203}$ Division of Newborn Medicine, Fetal Neonatal Neuroimaging and Developmental Science Center, Department of Pediatrics, Boston Children's Hospital, Boston, MA 02115

${ }^{204}$ McConnell Brain Imaging Center, Montreal Neurological Institute, McGill University, Montreal, Quebec, Canada

${ }^{205}$ Clinic for Cognitive Neurology, University of Leipzig Medical Center, Leipzig, 04103, Germany

${ }^{206}$ The Alan Turing Institute, London NW1 2DB, UK

${ }^{207}$ Wellcome Centre for Human Neuroimaging, Institute of Neurology, University College London, WC1N 3AR

${ }^{208}$ State Key Laboratory of Cognitive Neuroscience and Learning, Beijing Normal University, Beijing 100875, China

${ }^{209}$ Developmental Population Neuroscience Research Center, IDG/McGovern Institute for Brain Research, Beijing

Normal University, Beijing 100875, China

${ }^{210}$ National Basic Science Data Center, Beijing 100190, China

${ }^{211}$ Research Center for Lifespan Development of Brain and Mind, Institute of Psychology, Chinese Academy of

Sciences, Beijing 100101, China

${ }^{212}$ Division of Clinical Geriatrics, Center for Alzheimer Research, Department of Neurobiology, Care Sciences and

Society, Karolinska Institutet, Stockholm, Sweden

${ }^{213}$ Faculty of Medicine, CRC 1052 'Obesity Mechanisms', University of Leipzig, Leipzig, 04103, Germany

${ }^{214}$ Department of Electrical and Computer Engineering, National University of Singapore, Singapore

${ }^{215}$ Centre for Sleep \& Cognition and Centre for Translational MR Research, Yong Loo Lin School of Medicine, National University of Singapore, Singapore

${ }^{216}$ N.1 Institute for Health \& Institute for Digital Medicine, National University of Singapore, Singapore

${ }^{217}$ Integrative Sciences and Engineering Programme (ISEP), National University of Singapore, Singapore

${ }^{218}$ Fetal Neonatal Neuroimaging and Developmental Science Center, Division of Newborn Medicine, Boston

Children's Hospital, Harvard Medical School, Boston, MA 02115, USA

${ }^{219}$ Melbourne Neuropsychiatry Centre, University of Melbourne, Melbourne, Australia; Department of Biomedical

Engineering, University of Melbourne, Melbourne, Australia.

${ }^{220}$ SAMRC Unit on Child \& Adolescent Health, University of Cape Town, South Africa

${ }^{221}$ Center for Translational Magnetic Resonance Research, Yong Loo Lin School of Medicine, National University of Singapore, Singapore

${ }^{222}$ Wellcome Trust-MRC Institute of Metabolic Science, University of Cambridge, Cambridge, CB2 OSZ

${ }^{223}$ Cambridgeshire and Peterborough Foundation Trust, Cambridge, CB21 5EF

${ }^{224}$ National Institute of Mental Health (NIMH), National Institutes of Health (NIH), Bethesda, Maryland, USA

${ }^{225}$ Department of Psychiatry, Escola Paulista de Medicina, São Paulo, Brazil.

${ }^{226}$ Key Laboratory of Brain and Education, School of Education Science, Nanning Normal University, Nanning 530001, China

* Data used in this article were obtained from the brain consortium for reproducibility, reliability and replicability (3RBRAIN).

** Data used in the preparation of this article was obtained from the Australian Imaging Biomarkers and Lifestyle flagship study of ageing (AIBL) funded by the Commonwealth Scientific and Industrial Research Organisation (CSIRO) which was made available at the ADNI database (www.loni.usc.edu/ADNI). The AIBL researchers contributed data but did not participate in analysis or writing of this report. AIBL researchers are listed at www.aibl.csiro.au. 
*** Data used in preparation of this article were obtained from the Alzheimer's Disease Neuroimaging Initiative (ADNI) database (adni.loni.usc.edu). As such, the investigators within the ADNI contributed to the design and implementation of ADNI and/or provided data but did not participate in analysis or writing of this report. A complete listing of ADNI investigators can be found at: http://adni.loni.usc.edu/wpcontent/uploads/how_to_apply/ADNI_Acknowledgement_List.pdf

${ }^{* * * *}$ A complete listing of ARWiBo researchers can be found in the Supplementary Materials

***** Data used in this article were collected and processed by the UMN Baby Connectome Project (BCP) consortium and the Masonic Institute for the Developing Brain (MIDB) Informatics group. The groups are comprised of the following individuals: Amanda Ruetger, Audrey Houghton, Ben Lynch, Thomas Pengo, Jim Wilgenbusch, Tim Hendrickson, Trevor Day, Sooyeon Sung, Kathy Snider, Lucille Moore, Alice Graham, Damien Fair, Eric Feczko and Jed Elison

****** The Centre for Attention Learning and Memory (CALM) research clinic is based at and supported by funding from the Medical Research Council Cognition and Brain Sciences Unit, University of Cambridge, as well as funding from the EU Horizon 2020 Personalised Medicine "LifeBrain" project (H2020-SC1-2016-2017, Topic SC1-PM-042016). The lead investigators are Duncan Astle, Kate Baker, Susan E. Gathercole, Joni Holmes, Rogier A. Kievit, and Tom Manly. Data collection was assisted by a team of researchers and PhD students that includes Danyla Akarca, Joe Bathelt,Giacomo Bignardi, Sarah Bishop, Erica Botanic, Lara Bridge, Diandra Bkric, Annie Bryant, Sally Butterfield, Elizabeth Byrne, Gemma Crickmore, Edwin Dalmaijer; Fánchea Daly, Tina Emery, Grace Franckel, Laura Forde, Delia Fuhrmann, Andrew Gadie, Sara Gharooni, Jacalyn Guy, Erin Hawkins, Agniezska Jaroslawska, Sara Joeghan, Amy Johnson, Jonathan Jones, Elise Ng-Cordell, Sinéad O'Brien, Cliodhna O'Leary, Joseph Rennie, Ivan Simpson-Kent, Roma Siugzdaite, Tess Smith, Stepheni Uh, Francesca Woolgar, Mengya Zhang, and Natalia Zdorovtsova. We thank the many professionals working in children's services in the southeast and east of England for their support and to the children and their families for giving up their time to visit the clinic, and the radiographer for facilitating pediatric scanning.

******* The Cambridge Centre for Ageing and Neuroscience (Cam-CAN) research was supported by the Biotechnology and Biological Sciences Research Council (BB/H008217/1), as well as funding from the Medical Research Council Cognition and Brain Sciences Unit, and the EU Horizon 2020 Personalised Medicine "LifeBrain" project (H2020-SC1-2016-2017, Topic SC1-PM-04-2016). We thank the Cam-CAN respondents and their primary care teams in Cambridge for their participation in this study. Further information about the Cam-CAN corporate authorship membership can be found at http://www.cam-can.org/index.php?content=corpauth\#12.

******** Data used in this article were obtained from the developmental component 'Growing Up in China' of Chinese Color Nest Project (http://deepneuro.bnu.edu.cn/?p=163).

********* Data was downloaded from the COllaborative Informatics and Neuroimaging Suite Data Exchange tool (COINS; http://coins.mrn.org/dx) and data collection was performed at the Mind Research Network, and funded by a Center of Biomedical Research Excellence (COBRE) grant 5P20RR021938/P20GM103472 from the NIH to Dr. Vince Calhoun.

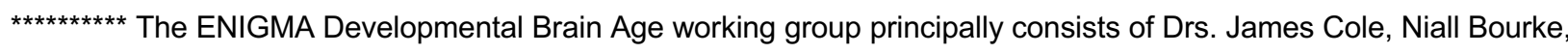
Heather Whalley, David Glahn, Laura Han, Francesca Biondo, Katherine Karlsgodt, Carrie Bearden, Jakob Seidlitz, Richard Bethlehem, Eileen Xu, Marieke Bos, Sam Mathia, Sophia Frangou, Miruna Carmen Barbu, Yoonho Chung, and Aaron Alexander-Bloch $* * * * * * * * * * *$ Data used in the preparation of this article were obtained from the Harvard Aging Brain Study (HABS P01AG036694; https://habs.mgh.harvard.edu). The HABS study was launched in 2010, funded by the National Institute on Aging. and is led by principal investigators Reisa A. Sperling MD and Keith A. Johnson MD at Massachusetts General Hospital/Harvard Medical School in Boston, MA.

$* * * * * * * * * * * *$ Data used in the preparation of this article were obtained from the IMAGEN consortium.

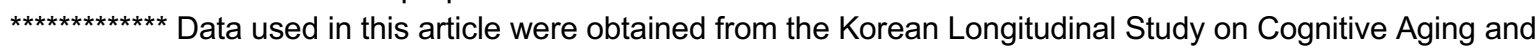
Dementia (KLOSCAD) (https://recode.re.kr).

${ }_{* \star \star * \star * \star * \star * \star \star * *}$ A full list of NSPN consortium members can be found at: https://www.nspn.org.uk/nspn-team/

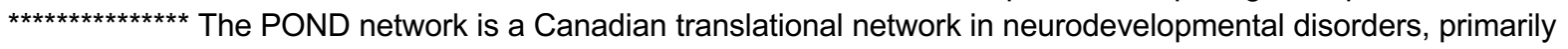
funded by the Ontario Brain Institute. 